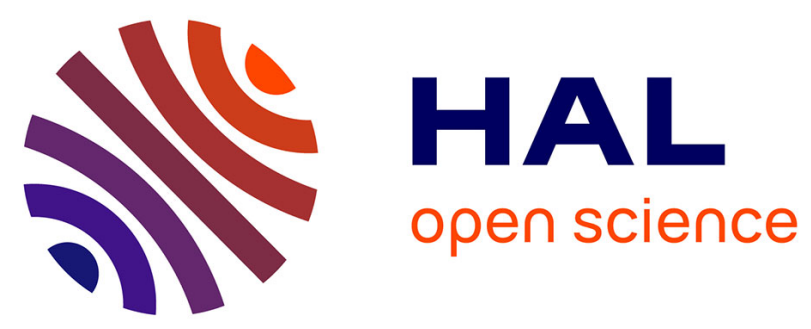

\title{
Space Charge at Nanoscale: Probing Injection and Dynamic Phenomena Under Dark/Light Configurations by Using KPFM and C-AFM
}

Christina Villeneuve-Faure, Kremena Makasheva, Laurent Boudou, G. Teyssedre

\section{To cite this version:}

Christina Villeneuve-Faure, Kremena Makasheva, Laurent Boudou, G. Teyssedre. Space Charge at Nanoscale: Probing Injection and Dynamic Phenomena Under Dark/Light Configurations by Using KPFM and C-AFM. Celano U. Electrical Atomic Force Microscopy for Nanoelectronics, Springer, pp.267-301, 2019, NanoScience and Technology, 10.1007/978-3-030-15612-1_9 . hal-02324281

\section{HAL Id: hal-02324281 \\ https://hal.science/hal-02324281}

Submitted on 1 Nov 2019

HAL is a multi-disciplinary open access archive for the deposit and dissemination of scientific research documents, whether they are published or not. The documents may come from teaching and research institutions in France or abroad, or from public or private research centers.
L'archive ouverte pluridisciplinaire HAL, est destinée au dépôt et à la diffusion de documents scientifiques de niveau recherche, publiés ou non, émanant des établissements d'enseignement et de recherche français ou étrangers, des laboratoires publics ou privés. 


\title{
Space charge at nanoscale: probing injection and dynamic phenomena under dark/light configurations by using KPFM and C-AFM
}

\author{
C. Villeneuve-Faure, K. Makasheva, L. Boudou, G. Teyssedre \\ LAPLACE, Université de Toulouse, CNRS, UPS, INPT, Toulouse, France
}

\section{Context}

Fine description of the electrical properties of solids at their surfaces is a very old problem, difficult to tackle because the surface of a solid itself represents a break in the periodic structure of crystallized materials, hence a defect, and most importantly because of the potential impact of surface oxidation, contamination, humidity, atmosphere, etc., on the material response [1]. For dielectrics, electrical charging of the surface leads to the build-up of a surface potential. The occurring mechanisms depend on the kind of charges being deposited, e.g. by triboelectrification, and are particularly difficult to anticipate [2-3]. Aside these difficulties in defining the surface properties, nanosciences and nanomaterials have brought us new paradigms with the tremendous increase of the amount of interfaces between particles and host matrix, and with the variety in the material nature and interface linked to the different elaboration processes. In a way it may constitute a chance to better describe what interfaces on an electronic properties standpoint are, because materials are better controlled. Besides the nanostructuration of materials, the miniaturization of devices is a further challenge to face. When dealing with thin layers (thicknesses of less than $100 \mathrm{~nm}$ ) the rules for bulk properties behavior are broken. Obviously, in both cases the experimental approach is more demanding, since the tools that are implemented for the study must have a spatial resolution compatible with the scale at which phenomena should be probed. In this Chapter we illustrate on a few examples the need for ever lower scale characterization of the electrical properties of dielectric materials.

\subsection{Miniaturization}

The specifications from the semiconductor technology sector are particularly demanding for what concerns gate dielectrics for transistors, with equivalent oxide thickness (EOT) of less than $1 \mathrm{~nm}$ in applications such as microprocessors (MPUs), or with low leakage current combined with EOT of less than $2 \mathrm{~nm}$ in applications such as cell phones, where the static power consumption is the major limiting factor [4]. This is an example of the break of bulk properties where the leakage current is through tunneling across the layer. To overcome this issue for gate dielectrics, high-k dielectrics are searched for replacement of the relatively low permittivity value of $\mathrm{SiO}_{2}$, with both fundamental (intrinsic material properties) and manufacturing limitations.

In the era of miniaturization, many benefits are being brought by Micro-ElectroMechanical Systems (MEMS), and now Nano-ElectroMechanical Systems (NEMS), to the downsizing to nanoscale, to exploit their capabilities in device size reduction, in response speed, energy conservation, autonomy, integration of complexity with combining sensors, actuators, energy harvesting, etc. There seems to exist a limitless possibility in MEMS-NEMS development. Because of their complexity, and of the necessity of motion of piece parts, the MEMS-NEMS are naturally exposed to ambient stresses such as vibration, humidity or pollution, which makes their reliability an issue [5]. The stresses endowed by materials can be relatively strong, an example being of electrostatic origin due to the charging of materials that can lead to malfunction of voltage-driven actuators. Though driving voltages are relatively small, the electric fields are high and the device geometry often imposes high diverging field such as the charge deposition is highly effective. Another important issue for the reliability of MEMS is tribological effect particularly appearing on the contacts [6]. For probing devices with such 
outcomes, appropriate techniques and methodologies are needed to provide information on charges and forces at the relevant scale [7-9].

Whereas the charge trapping represents an issue for MEMS devices, it is exploited in non-volatile memory devices to store the information. Here again the objectives are to miniaturize and to improve reliability. Indeed, in order to keep up with the demand for increased memory capacities, flash memory devices have been continuously scaled down. The main benefits of down-scaling the cell size and increasing integration are enabling lower manufacturing cost while keeping high performance. Charge trapping memory is regarded as one of the most promising flash memory technologies at further down-scaling strategies. High-k dielectrics are usually preferred as charge trapping layer, blocking layer, and tunneling layer [10]. $\mathrm{SiO}_{2}$ is the first dielectric used as blocking layer in the flash memory. However, as for transistors, the large tunneling current through $\mathrm{SiO}_{2}$ is not acceptable upon continually scaling down the dimensions of the flash memory.

\subsection{Interfaces}

In organic solar cells, the low diffusion length of excitons imposes that donor and acceptor materials segregate to form small size domains with high interface area to reach reasonable efficiency. To optimize the efficiency, intermixing of the donor and the acceptor moieties on the nanometer scale is essential. This insight led to the development of the so-called bulk-heterojunction concept [11]. Connected domains with a typical size of several tens of nanometers are formed in the film. At the same time a small amount of the acceptor material may be dissolved in the donor domains or vice versa. Obviously, control and optimization of such structures require adequate tools [12].

Parameters of outmost value, when dealing with interfaces, are the energy level estimations of one material relative to the other one as they determine the working conditions of the device. When going to thin films, these energy levels become modified and very new properties are being introduced with the use of 2D heterostructures made of few atom thick layers [13]. Besides the physical properties of materials and junctions, the behavior of materials under stress should also be evaluated. With the new tools brought by scanning probe techniques, Atomic Force Microscopy (AFM) in particular, it becomes now possible to evaluate in details the mechanisms at the origin of charges generation into dielectrics, and to assess and revise the models for charge injection currently under use [14-15]. Moreover, it is important to characterize how such charges are dissipated in the bulk or at the surface.

Specific dielectric properties of nanocomposite materials, made of nanoparticles (not necessarily insulating) dispersed into insulating matrices have been recently reported [13, 16]. The claimed effect has been associated with the structuration of the matrix under the impact of the nanoparticles or to "charge trap" formation at the interfaces [17]. It has also been shown that differences imposed by the polymer dielectric interface processing can lead to substantial changes in the macroscopic response of the material [18]. However, future developments should contribute to assess the different hypotheses put behind changes in the charging behavior of bulk materials.

The above given examples are only a flavor of how broad can be the field of electronic properties and electrical charges profiling into materials. Certainly many other aspects could be addressed. Our purpose in the following is to present newly developed methods likely to provide information at pertinent scale to address these problems of electrostatics: local information is needed either because the investigated process is actually at the interface like for charge injection or energy levels estimation, or because the structures have been downsized and existing methodologies to probe the properties cannot be easily adapted. This is for example the case of charge density distribution measurements. 


\section{KPFM and C-AFM measurement under dark and light configurations}

\subsection{Introduction to surface potential}

In electrochemistry the surface potential in a solid is classically defined as the difference between the internal and the external electric potentials i.e., Galvani and Volta potentials, respectively [19]. Consequently, it depends on the material properties (crystallographic structure...) and the surface features (adsorbed molecules...) and for the different materials is defined as follow: For a metal (Fig.1.a), the surface potential therefore corresponds to the work function $\phi_{\mathrm{m}}$ which is the amount of energy required to extract an electron from fundamental state in the material to the vacuum level [20]. For a semiconductor, the surface potential corresponds to the energy of Fermi level (Fig.1.b). It is influenced by the interface states, which induce band curvature, and by the level of doping [21]. Indeed, n-doping type decreases the Fermi level and consequently, the surface potential (Fig.1.c). For an insulator, the definition of surface potential is scarcely reported in the literature. However, the surface potential reflects the charge distribution present in the insulation [22]. In the presence of electrons (holes) the surface potential is decreased (increased). So, as for the semiconductors, the surface potential should be considered as the energy difference between the vacuum level and the last energy level under the conduction band (CB).
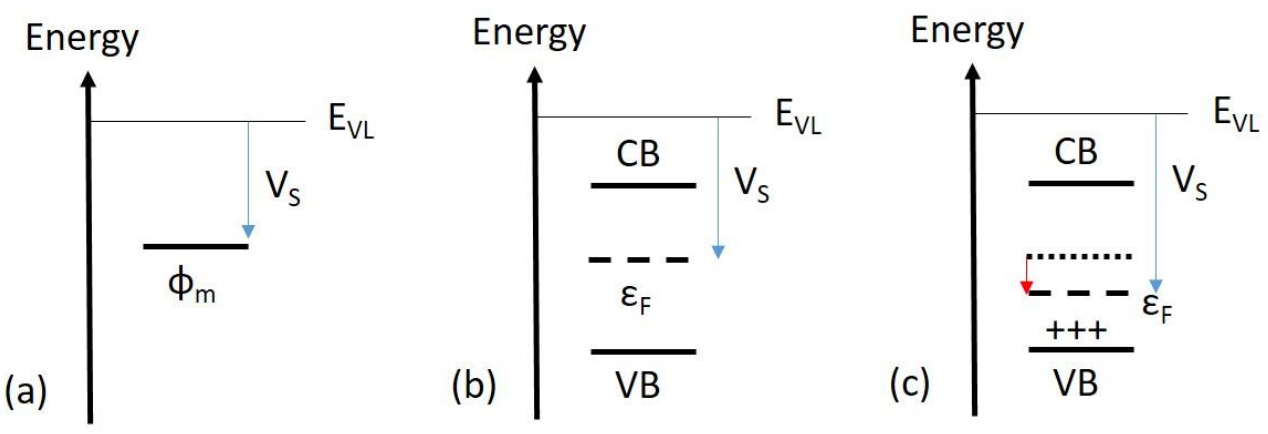

Fig.1. Energy level of (a) metal and (b) semiconductor or dielectric material with the corresponding surface potential $V_{S}$. (c) In presence of doping (semiconductor) or charges (dielectric) the surface potential is modified. Positive charges induce surface potential increase. $\varepsilon_{\mathrm{F}}$ is the Fermi level. $\mathrm{CB}$ and $\mathrm{VB}$ are the conduction and valence bands, respectively. $\mathrm{E}_{\mathrm{VL}}$ is the vacuum level.

\subsection{Surface potential measurement in AM-KPFM}

Kelvin Probe Force Microscopy (KPFM) permits to probe the surface potential difference between an AFM tip and the sample surface. This difference, named $\Delta \phi$, corresponds to Contact Potential Difference $\mathrm{V}_{\mathrm{CPD}}$ which is expressed by:

$$
\Delta \phi=\mathrm{V}_{\mathrm{CPD}}=\phi_{\mathrm{m}}-\mathrm{V}_{\mathrm{S}},
$$

where $\phi_{\mathrm{m}}$ is the work function of the conductive AFM probe and $\mathrm{V}_{\mathrm{S}}$ is the sample surface potential. To enable surface potential measurements a lot of different KPFM modes were developed during the past decade. In this section, Amplitude Modulation KPFM (AM-KPFM) is presented to introduce the principles of surface potential measurement and their limitations.

\subsubsection{Principles}

The AM-KPFM is performed in lift mode, that is to say the measurement runs in two steps. During the first pass, surface topography is acquired in tapping mode (with mechanical oscillation of the AFM probe at its resonance frequency $\omega_{0}$ ). During the second pass, the tip is moved away from the surface by a fixed height, $\mathrm{h}$ (called lift height) and the mechanical oscillation at $\omega_{0}$ is stopped. Instead, a voltage $\mathrm{V}_{\text {bias }}$ is applied to the AFM probe constituted by a DC component $\mathrm{V}_{\mathrm{dc}}$ and an AC component $\mathrm{V}_{\mathrm{ac}}$ at the same pulsation $\omega_{0}$ as the mechanical oscillations:

$$
\mathrm{V}_{\text {bias }}=\mathrm{V}_{\mathrm{dc}}+\mathrm{V}_{\mathrm{ac}} \sin \left(\omega_{0} \mathrm{t}\right)
$$


During KPFM measurement, the AFM probe scans the material surface at lift height and the surface potential difference $\Delta \phi$ is superimposed to the applied bias. For $\mathrm{V}_{\text {bias }}$ applied on the tip, an electrostatic force $\mathrm{F}_{\mathrm{e}}$ is induced on the AFM probe that depends on the sample tip distance z. [23]

$$
\mathrm{F}_{\mathrm{e}}=-\frac{1}{2} \frac{\mathrm{dC}}{\mathrm{dz}}\left(\mathrm{V}_{\mathrm{dc}}-\Delta \phi+\mathrm{V}_{\mathrm{ac}} \sin \left(\omega_{0} \mathrm{t}\right)\right)^{2}
$$

with $\mathrm{C}$ being the capacitance formed by the AFM probe and the sample. The electrostatic force can be split into three components:

with

$$
\mathrm{F}_{\mathrm{e}}=\mathrm{F}_{\mathrm{DC}}+\mathrm{F}\left(\omega_{0}\right)+\mathrm{F}\left(2 \omega_{0}\right)
$$

$$
\begin{aligned}
& \text { the DC component: } \mathrm{F}_{\mathrm{DC}}=\frac{1}{2} \frac{\mathrm{dC}}{\mathrm{dz}}\left(\left(\Delta \phi-\mathrm{V}_{\mathrm{dc}}\right)^{2}+\frac{\mathrm{V}_{\mathrm{ac}}^{2}}{2}\right. \\
& \text { the } \omega_{0} \text {-component: } \mathrm{F}\left(\omega_{0}\right)=-\frac{\mathrm{dC}}{\mathrm{dz}}\left(\Delta \phi-\mathrm{V}_{\mathrm{dc}}\right) \mathrm{V}_{\mathrm{ac}} \sin \left(\omega_{0} \mathrm{t}\right) \text {, } \\
& \text { the } 2 \omega_{0} \text {-component: } \mathrm{F}\left(2 \omega_{0}\right)=\frac{1}{4} \frac{\mathrm{dC}}{\mathrm{dz}} \cdot \mathrm{V}_{\mathrm{ac}}^{2} \cos \left(2 \omega_{0} \mathrm{t}+2 \varphi\right)
\end{aligned}
$$

In AM-KPFM mode, the $\mathrm{V}_{\mathrm{DC}}$ bias is adjusted as to cancel the electrostatic force at $\omega_{0}$. It is determined at each point and corresponds to the potential difference $\Delta \phi$ between the tip and the surface of the sample. Using this mode, it is therefore possible to probe simultaneously surface topography and potential difference between the tip and the surface.

\subsubsection{AM-KPFM performances and limitations}

According to the literature, the smallest surface potential value measured by AM-KPFM is of $5 \mathrm{mV}$ [24]. It is more difficult to determine a common lateral resolution for all kinds of samples. Indeed, the lateral resolution depends on several parameters [24-25] such as the AFM tip curvature radius, the lift height, the surface topography, the nature of material, etc. The lateral resolution is estimated between $10 \mathrm{~nm}$ and 100nm [26-27]. However, it is mainly limited by three parasitic contributions: (i) measurement environment, (ii) probe contribution due to parasitic capacitance and (iii) topography. These effects are detailed in the following:

- Environment

KPFM measurements in air environment exhibit the worst resolution and the lowest potential sensitivity. This is mainly due to the water layer formation on the sample surface. Measurements performed under controlled environment (dry air or $\mathrm{N}_{2}$ atmosphere) prevent from water layer formation and improve KPFM performances. However, measurements done under vacuum present the best spatial resolution and the highest sensitivity due to quality factor improvement and sample surface cleanness. So, this configuration is required for absolute surface potential measurements. Moreover, under vacuum, the surface topography is measured no more in tapping mode but in Non-Contact mode [28]. The later was extensively used to investigate thin dielectric films and managed to reach atomic resolution [29-30].

\section{- $\quad$ KPFM tip contribution}

Parasitic capacitance is the main issue during surface potential measurement by KPFM. Indeed, as shown on Fig. 2, surface features localized close to the measurement point (here a spot) contribute to the KPFM signal. Jacobs et al. [24] demonstrated that the parasitic capacitance reduces the measured potential and deteriorates the lateral resolution due to averaging effects. This phenomenon is amplified when the lift height is increased. To determine the real potential from the KPFM measurements, an approach based on deconvolution of the measured potential profile by a Gaussian type function was proposed [31]. This function, called Point Spreading Function (PSF) of the system represents the transfer function of the AFM probe. The Full-Width at Half maximum (FWHM) and the maximum of the PSE are calculated for given height of the lift and for curvature radius of the AFM probe [32].

Table I. PSF FWHM and maximum (peak) computed for AFM probe with curvature radius of 30nm for different lift heights in AM and FM mode [32]. 


\begin{tabular}{|c|c|c|c|c|c|c|c|}
\hline \multicolumn{2}{|c|}{ Lift height (nm) } & 2 & 5 & 10 & 20 & 50 & 100 \\
\hline $\begin{array}{c}\text { AM- } \\
\text { KPFM }\end{array}$ & $\begin{array}{c}\text { PSF } \\
\text { HWHM } \\
\text { (nm) }\end{array}$ & 9 & 20 & 33 & 48 & 82 & 165 \\
\cline { 2 - 8 } & PSF peak & $1.810^{-3}$ & $310^{-4}$ & $110^{-4}$ & $4.510^{-5}$ & $710^{-6}$ & $1.510^{-6}$ \\
\hline $\begin{array}{c}\text { FM- } \\
\text { KPFM }\end{array}$ & $\begin{array}{c}\text { PSF } \\
\text { HWHM } \\
\text { (nm) }\end{array}$ & 6 & 17 & 25 & 35 & 65 & 110 \\
\cline { 2 - 8 } & PSF peak & $810^{-2}$ & $110^{-3}$ & $710^{-4}$ & $310^{-4}$ & $110^{-4}$ & $310^{-5}$ \\
\hline
\end{tabular}

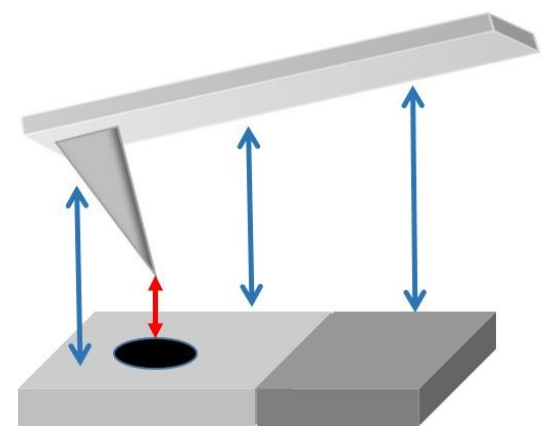

Fig. 2. Contribution of parasitic capacitance (blue arrow) compare to main contribution (red arrow) during KPFM measurement over a spot.

- Topography influence

The crosstalk between topography and surface potential mapping is mainly related to the $\mathrm{dC} / \mathrm{dz}$ contribution in eq. 6 . For samples with flat surfaces, presenting roughness of only few nanometers, the contribution due to the crosstalk remains in the same range as the spatial resolution and the topography influence are quite small. However, topography features such as steps and grooves are often present. Zerweck et al demonstrated that the real magnitude of a potential step is not reached for distances as large as $500 \mathrm{~nm}$ away from the step [27]. To weigh this influence, Sadewasser et al have shown that step and groove topography has different contributions to the potential peak/step (Table II) [33]. Moreover, this effect is strengthened when increasing lift height and tip oscillation amplitude during potential measurements [34]. Consequently, these two parameters should be maintained as small as possible to reach the best spatial resolution and to avoid artefacts. So, even if the AM-KPFM presents some drawbacks, it remains the most used KPFM mode.

Table II. Influence of step and groove topography on potential peak/step profile

\begin{tabular}{|c|c|c|}
\hline Potential peak & Small topography step & Groove topography \\
\hline Potential step & $\begin{array}{c}\text { Increase of the peak width } \\
\text { Peak maximum remains } \\
\text { constant }\end{array}$ & Maximum is sligthly decreased \\
\hline $\begin{array}{c}\text { Potential step shifts toward } \\
\text { lower terrace }\end{array}$ & No general effect \\
\hline
\end{tabular}

\subsection{Surface potential measurement in FM-KPFM}

Another common mode for surface potential measurements is Frequency Modulation KPFM (FMKPFM). In this mode, the surface potential can be measured in lift [35] or single pass [27, 36] configurations. The single pass mode permits to probe topography and surface potential simultaneously. In this mode, the frequency of AC excitation $\omega$ is lower than the mechanical resonance frequency $\omega_{0}$. For single pass-measurement, this difference is exploited to discriminate topographical and surface potential contributions.

Contrary to AM-KPFM, the FM-KPFM close-loop tunes $V_{D C}$ to null force gradient at $\omega$, which is linked to the frequency shift $\Delta f_{\omega}$ by the following relation: 


$$
\Delta f_{\omega} \propto \frac{d F(\omega)}{d z}=-\frac{d^{2} C}{d z^{2}}\left(\Delta \phi-V_{d c}\right) V_{A C} \sin (\omega t) .
$$

The use of the force gradient allows better spatial resolution and measurement of the surface potential close to the real one. Indeed, this effect is confirmed by the corresponding PSF parameters that present lower FWHM and higher amplitude than the AM ones (Table I). Moreover, the FM-KPFM mode has lower noise level compared to the AM-KPFM, thus leading to an increase of the sensitivity and allowing detection of smaller surface potential [35].

Relative merit of the AM and FM modes was studied in details by Meliz et al [28] and Ziegler et al [35]. In summary, the spatial resolution of $\mathrm{V}_{\mathrm{CPD}}$ when measuring in FM-KPFM mode is higher than in AM-KPFM mode. In contrary, the energy resolution of $\mathrm{V}_{\mathrm{CPD}}$ in measurements under FM-KPFM mode is lower than in AM-KPFM mode. However under AM-KPFM mode the $\mathrm{V}_{\mathrm{CPD}}$ is measured from the resonance peak of the oscillating cantilever which greatly enhances the signal-to-noise ratio.

\subsection{Surface potential measurement in PF-KPFM}

Peak Force-KPFM (PF-KPFM) is a quite new mode introduced in 2010 by Bruker ${ }^{\circledR}$. It combines the advantages of Peak Force Quantitative NanoMechanical (PF-QNM) mode and the high spatial resolution of FM-KPFM [37]. The PF-KPFM is performed in lift mode in two measurements steps:

(i) Topography is measured in PF-QNM which allows improvement of the lateral resolution, decrease of the interaction force while probing simultaneously surface mechanical properties [38].

(ii) Surface potential is measured in lift mode using FM-KPFM.

The main advantages of PF-KPFM are related to the AFM probe which is specially designed with high quality factor $\mathrm{Q}$, low spring constant $\mathrm{k}$ and without coating (very smal tip radius). As a consequence the surface potential profile can be probed by PF-KPFM with high spatial resolution and with close to the theoretical $\mathrm{V}_{\mathrm{CPD}}$ values [37]. However, until now this mode is dedicated to surface potential mapping and does not appear suitable to investigate charge distribution in dielectric films. Indeed, a strong limitation of the method seems to be the repetitive contact of the AFM tip that can modify the charge distribution during contact step in PF-QNM topography measurement.

\subsection{Photoconductive and photo-KPFM modes}

Essentially three different current measurement methods using a conductive AFM tip have been developed depending on the available current range:

(i) Scanning Spreading Resistance Microscopy (SSRM) which uses logarithmic amplifier to probe current in the range from $100 \mathrm{nA}$ to $100 \mu \mathrm{A}$. This is an indirect current measurement method because an abacus is needed to convert the measured bias to resistance [39].

(ii) Conductive AFM (C-AFM) that uses linear amplifier to probe current in the range from 100 pA to $100 \mathrm{nA}$.

(iii) Tunneling AFM (TUNA) which is a mode derived from the C-AFM and uses a low noise linear amplifier to probe current at lower range from 50fA to around 100pA [40].

From a general point of view the current measurements are obviously performed in contact mode. In this configuration the contact force between the AFM tip and the sample surface is a crucial parameter. Indeed, this force is determined as compromise between a high force to ensure reliable mechanical contact and a low force to avoid tip degradation (coating damage, tip radius increase, etc.).

Recently, in the attempt to find the best compromise for the contact force, a mode using Peak-Force technique named PF-TUNA was developed [41]. In this mode the current is probed in different configurations: Peak Current (current at the maximum contact force), Contact average Current (current mean value over the entire contact phase) and Cycle average Current (current mean value over the entire approach/retract process). Other advantage of this mode is to probe simultaneously surface 
topography, mechanical properties and electrical current. However, a comparison between the C-AFM and PF-TUNA revealed that higher contact force is required in PF-TUNA than in C-AFM to reach the same current value [42].

In some applications as solar cells or water-splitting photochemical cells, light is one of the main components of the system. Accordingly, to understand the influence of the active layer properties under illumination on the device performance, a light source was added to the AFM set-up to probe electrical current under dark and light conditions. Fig. 3 represents a classical AFM set-up modified to provide sample backside illumination, thus allowing realization of electrical measurements (mainly CAFM, KPFM and EFM) under light conditions [43]. Various light sources have been implemented to evaluate the measurement procedure: laser with accordable wavelength, reverse microscope and solar cell simulator. Independently on the light source, the light power density should be controlled accurately to avoid thermal effects that can induce abnormal carrier conduction or thermal expansion leading to an increase of the contact force between tip and surface [44].

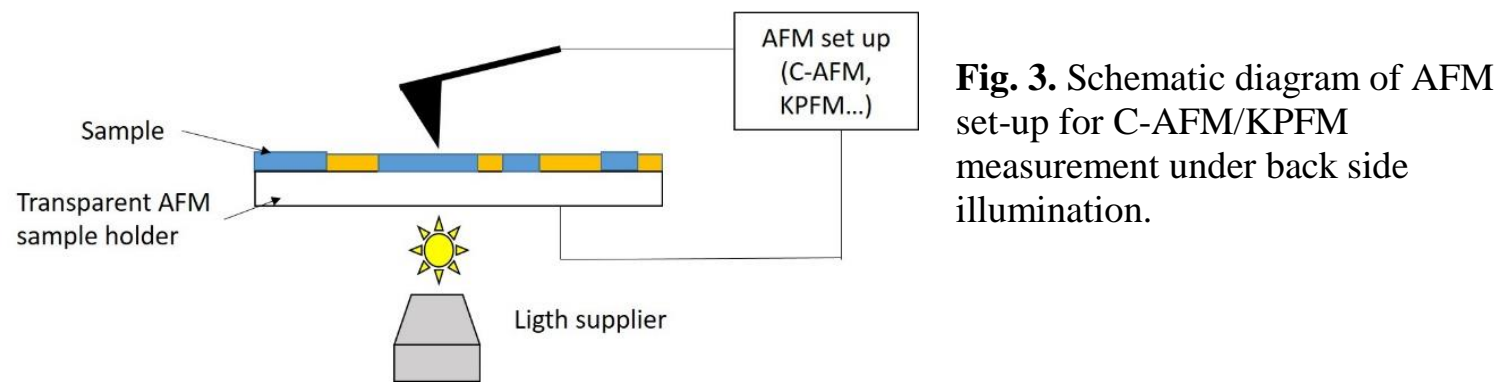

\subsection{KPFM modelling}

The modelling of the electrostatic force and potential produced by interaction of the AFM tip and the sample surface faces the problem of strongly divergent field and requires multiscale approach. Therefore, simplifying hypotheses have been considered for the geometry. The aim of the modelling is multifold: first it serves as optimization tool for operating conditions and design of the instrument; second it may help identifying artefacts from useful signal and finally, it could help, in a reverse form to reach the charge distribution providing a given KPFM signal.

\subsubsection{Electrostatic force modelling}

The electrostatic force $\mathbf{F}_{\mathbf{e}}$ between the AFM tip and sample surface can be expressed by the following equation:

$$
\mathbf{F}_{\mathbf{e}}=\frac{\varepsilon_{0}}{2} \iint_{\text {Sonde }}\|\mathbf{E}\|^{2} \boldsymbol{d} \mathbf{S}
$$

with $\mathrm{E}$ the electric field, $\varepsilon_{0}$ is the dielectric permittivity and $\mathrm{S}$ in the sample surface.

According to the literature, different methods to compute the electrostatic force applied on the AFM tip were developed.

- Image charge model [45];

- Analytical model which computes the electrostatic force for each part of the AFM tip: sphere (tip apex), cone (tip main part) and cantilever [46]. Results provided by this approach present a good agreement with experimental ones for short tip-sample distances;

- Finite element model (FEM) reproducing the AFM tip in 2D axisymmetrical geometry [4748]. This model is typically used for interpretation of KPFM measurements;

- 3D FEM reproducing the real pyramidal AFM-tip shape [49]. This approach proposed recently, exhibits a good agreement with experimental results also for large tip-sample distances but requires longer computational times. 


\subsubsection{Surface potential modelling}

In the current state, two different models were developed to reproduce KPFM measurements:

- Standard model. The electrostatic force is computed using one of the methods described above as a function of the bias applied on the tip $\mathrm{V}_{\mathrm{DC}}$. The relationship between the electrostatic force and the $\mathrm{V}_{\mathrm{DC}}$ is of parabolic type with minimum occurring for the surface potential [50]. This model is commonly used even though it does not take into account the fact that the KPFM is not sensitive to image charge;

- Model proposed by L. Borowik et al., which considers the fact that the KPFM is insensitive to image charge [51]. The model accounts for side-capacitance and nonlinear effects taking place in the KPFM experiments. Concerning AM-KPFM, the surface potential $\mathrm{V}_{\text {Surf }}$ is expressed as follows:

$$
V_{S u r f}=-\frac{V_{D C}\left(F_{Q-V}-F_{Q}-F_{V}\right)}{2 F_{V}} .
$$

For a given applied bias $\mathrm{V}_{\mathrm{DC}}, \mathrm{F}_{\mathrm{Q}-\mathrm{V}}$ is the electrostatic force with charge $\mathrm{Q}$ and bias applied on tip, $F_{Q}$ is the electrostatic force with charge $\mathrm{Q}$ (image charge effect) and $\mathrm{F}_{\mathrm{V}}$ is the electrostatic force with applied bias $\mathrm{V}_{\mathrm{DC}}$ on tip.

A similar model was proposed for FM-KPFM by L. Borowik et al. [51].

\section{Local charges injection mechanisms}

Local charge injection and decay mechanisms, in thin dielectric layers are important issues due to their impact on the performances of a number of micro- and nano-devices. Indeed, even if the charge retention is exploited in non-volatile memories [52] for example, this phenomenon remains the main cause of failure in MicroElectroMechanical Systems [53] or CMOS devices with thin gate dielectric layers [54]. Consequently, charge injection and dynamics in thin active dielectric layers require characterization at local scale.

During the past decade, charge injection and retention were extensively studied locally using the electrical modes derived from AFM. First of all, the Electrostatic Force Microscopy (EFM) was employed [55-57]. However, the EFM sensitivity to image charge implies difficulties in the interpretation of the experimental results. Therefore, KPFM tends to be preferred to investigate charge injection and retention in thin dielectric films [58-59].

From an experimental point of view, the charge injection and decay studies appear strongly influenced by the way charges are generated and by the measurement conditions. These issues related to the charging method and the KPFM measurement constrains will be detailed in the following part before providing some illustration of the KPFM potentiality for investigation of charge injection and decay mechanisms. In the last part the remaining bottlenecks and ongoing developments will be presented.

\subsection{Local injection using conductive AFM-tip and surface potential measurements}

\subsubsection{Methodology for local charges injection using AFM tip}

Charge retention in dielectrics was extensively studied at macroscopic scale by using Corona gas discharge for charging (ions deposition) and Kelvin probe technique for macroscopic surface potential measurements [60-61]. In the early 90's, due to the AFM improvement, local charge injection using conductive AFM tip was developed. This approach was introduced by C. Schonenberger and based on triboelectrication as charging method [62]. In this configuration, the AFM tip is grounded and rubbed on the surface. Five years later, the charge injection in thin dielectric layers was performed using applied bias on a conductive AFM tip (Saint Jean et al 1997). Two configurations are available for charge injection with AFM tip either in contact or in tapping mode (Fig.4). In contact mode, a constant force $\mathbf{F}_{\mathbf{c}}$ is maintained between the AFM tip and the sample surface during all charging process. In tapping mode, the conductive AFM tip oscillates at fixed amplitude/frequency close to the dielectric 
surface. For charging, a bias is applied on the AFM tip. This can be dc or ac bias whose characteristics influence strongly the charging process. After charging, the following step consists in probing the resulting surface potential KPFM (Fig. 4). The experimental results show that the surface topography is not influenced by the charges injection (Fig. 5.a) whereas the surface potential is modified by the injected charges cloud (Fig. 5.b). During the charging process, the AFM tip can be moved over the dielectric surface resulting in a contour for the injected charges cloud. This process named electrostatic patterning is illustrated on Fig. 5.c and Fig. 5.d. In this example, charges are generated in tapping mode after applying ac-bias on the AFM-tip. So, the charges injection using AFM tip appears a complex process due to the combination of injection mode (contact vs. tapping), patterning (local or surface charging) and applied bias (dc vs. ac. The relative merit of each configuration is summarized in Table III.
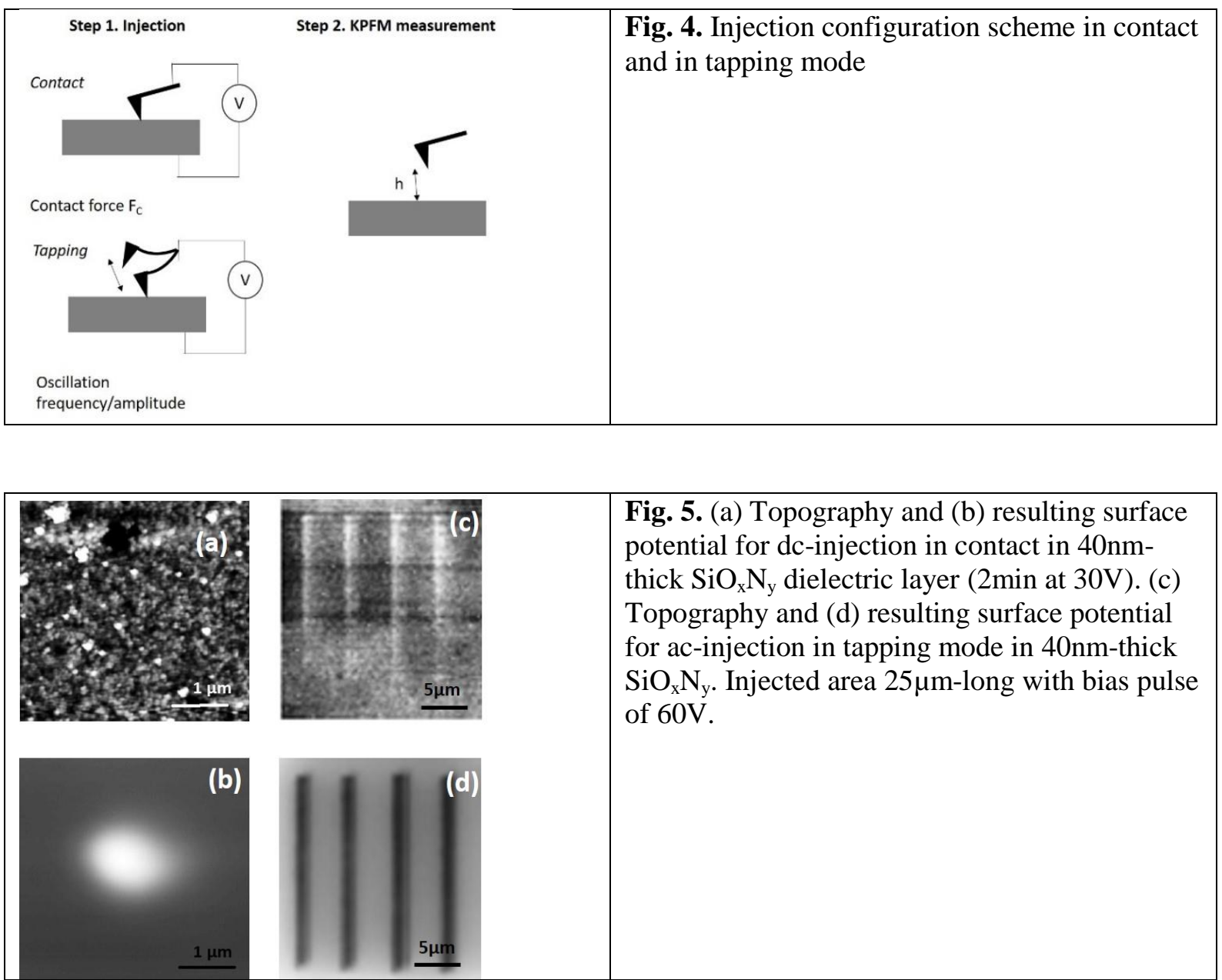

Table III. Comparison of advantages and drawbacks of different injection conditions

\begin{tabular}{|c|c|c|c|c|c|c|}
\hline & \multicolumn{2}{|c|}{ Injection mode } & \multicolumn{2}{|c|}{ Pattern } & \multicolumn{2}{|c|}{ Applied voltage } \\
\hline & Contact & Tapping & Point & Contour & DC & $\mathbf{A C}$ \\
\hline Advantages & $\begin{array}{l}\text { Reliable } \\
\text { injection } \\
\text { conditions }\end{array}$ & $\begin{array}{l}\text { Less tip } \\
\text { damage }\end{array}$ & $\begin{array}{l}\text { Simple } \\
\text { shape of } \\
\text { potential } \\
\text { profile }\end{array}$ & $\begin{array}{l}\text { Tunable } \\
\text { dimensions }\end{array}$ & $\begin{array}{l}\text { Reliable } \\
\text { injection } \\
\text { conditions }\end{array}$ & $\begin{array}{l}\text { Amount of } \\
\text { injected } \\
\text { charge higher } \\
\text { than DC }\end{array}$ \\
\hline Drawbacks & $\begin{array}{l}\text { Possible tip } \\
\text { damage }\end{array}$ & $\begin{array}{l}\text { Injection } \\
\text { conditions } \\
\text { roughly } \\
\text { evaluated }\end{array}$ & $\begin{array}{l}\text { Charged } \\
\text { area } \\
\text { depends } \\
\text { on } \\
\text { material }\end{array}$ & $\begin{array}{l}\text { Possible tip } \\
\text { damage }\end{array}$ & $\begin{array}{l}\text { Amount of } \\
\text { injected charge } \\
\text { weaker than } \\
\text { AC }\end{array}$ & $\begin{array}{l}\text { Possible } \\
\text { parasitic tip } \\
\text { oscillations } \\
\text { Charging } \\
\text { mechanism }\end{array}$ \\
\hline
\end{tabular}


According to the charge injection configuration three main approaches can be identified in the literature:

(i) Electro-triboelectrification which consists in applying DC-bias on the AFM tip brought in contact with dielectric layer and rubbed over it [63-64];

(ii) Charge injection in contact mode applying DC-bias on fixed AFM tip. This is mainly used to study punctual charge injection process with contact between the tip and the sample surface thus improving the injection configuration repeatability [65-66];

(iii) Electrostatic patterning configuration using tapping mode and DC-bias to produce injected charge pattern. This configuration is used for applications in nanolithography for nano patterning [67];

For punctual charge injection configuration (Fig. 5.b) the lateral cross-section of the potential profile exhibits a Gaussian shape (Figure 6). Three parameters characterizing the injected charge can be extracted from this experimental profile:

(i) Full-Width at Half Maximum (FWHM) which reflects the lateral spreading of the injected charge;

(ii) Surface potential maximum $\mathrm{V}_{\mathrm{m}}$ relative to the reference surface potential without charge;

(iii) Area under the potential profile $\mathrm{I}_{\mathrm{s}}$ which is supposed to represents an image of the amount of injected charge. Indeed, when the charges are located on the surface (or close to surface compared to tip radius) the area under the potential profile, i.e. $\mathrm{I}_{\mathrm{s}}$, is proportional to the amount of injected charge [68];

Moreover, these parameters can be used to provide information on the process of charge release, in particular to distinguish charge surface spreading and charge drift in the insulation bulk.

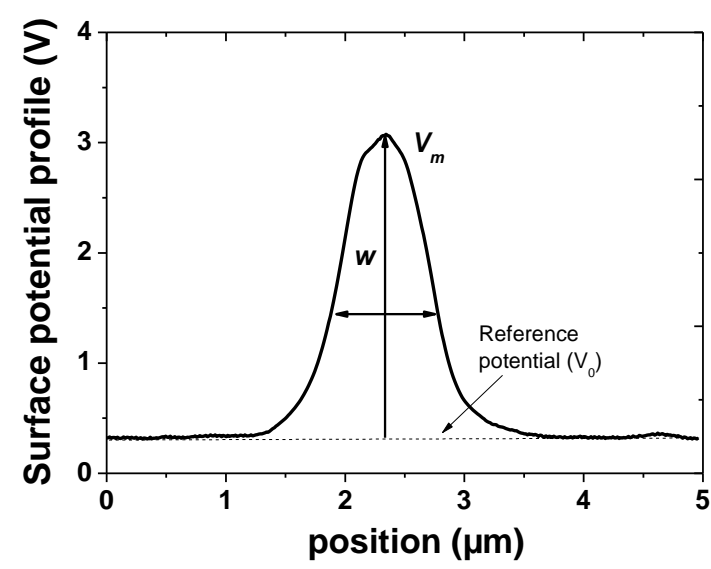

Fig. 6. Surface potential profile crosssection over contact point for punctual charge injection (2D surface potential map is depicted on Fig. 5.b).

3.5.2. Influence of the experimental conditions on the charges injection process Various studies emphasize that the experimental results provided by KPFM are very sensitive to the experimental conditions, such as nature of the tip, polarity and magnitude of the applied voltage, charging time, etc. These are parameters easy to control. So, they will not be involved in the analysis here. On the contrary, other subtler parameters have strong contribution to resulting surface potential as explained in the following.

\section{- Environmental conditions}

The influence of environmental conditions (humidity or ambient gas) on KPFM measurement after local charge injection depends strongly on the dielectric material under study. High humidity level in the measurement chamber induces a water layer build-up on the dielectric surface which creates a meniscus with the AFM tip. This phenomenon has different impacts: 

deposition in water layer instead of on the dielectric layer. In this case, the KPFM results reflect mainly charge dynamic in the water layer [7, 58];

(ii) Local oxidation due to chemical reaction in the water layer induced by the strong electric field. This effect results in modification of the surface topography which in general is not altered by the applied voltage on the tip during topography measurement [69],

(iii) No real impact mainly due to dielectric layer hydrophobic properties [70]. Moreover, Sridhara et al reported that in dry conditions, the ambient gas may impact charge injection and decay [71]. Consequently, strict control of the environmental conditions is essential for gathering reliable and reproducible results.

\section{- Apparent height effect}

When the amount of injected charge is large, the electrostatic force could parasite the AFM probe control during topography measurements. This induces a correlation between topography and surface potential mapping and an apparent height is observed on topography map as shown on Fig 5.c for high injection bias. Ziegler et al demonstrate that this effect can be reduced by applying bias during topography measurement [72]. As the topography is reproduced during measurement with KPFM in lift mode, this apparent height induces surface potential decrease which is not linked to the charge decay in the dielectric layer.

\subsection{Charges injection and decay in thin dielectric layers}

\subsubsection{Issue about charges injection mechanisms}

From an outlook of the literature, it can be realized that charges stabilization and release in thin dielectric layers were much more extensively studied than the injection mechanisms. The identification of mechanisms occurring during charges injection from metalized AFM-tip into dielectric layers is not straightforward. Indeed, the local character of mechanisms, the highly diverging nature of the electric field, its enhancement close to the tip and the small distance between tip and sample surface prevents the use of macroscopic models. However, this aspect is ill referenced in the literature. Up to now only few mechanisms were proposed to explain the charge injection in thin dielectric films by AFM. Historically, based on macroscopic approaches, injection by corona discharge was evoked as the main mechanism for dielectric charging by AFM [73]. However, recent theoretical calculations seconded by experimental demonstration, confirmed that corona discharge cannot develop in such small tip-to-sample distances [74]. Instead, the field electron emission enhanced by thermionic electron emission is proposed as the main mechanism.

Moreover, the influence of tip characteristics (geometry, work function of the coating...) is rarely considered. Sun et al. briefly addressed the influence of the material work function of the AFM tip on the charge injection, by comparing features from two tips with different coatings [75]. This was completed by C. Villeneuve-Faure et al, demonstrating that the work function of the metal coating of the tip influences carrier injection differently depending on the applied voltage polarity [14]. Indeed, electrons injection follows the Schottky barrier law whereas holes seem to be insensitive to the injection barrier height. Holes injection would be mainly driven by interface states. These aspects remain to be revised after deeper investigation.

\subsubsection{Charges injection and decay}

According to Morita et al, when charges are stored locally on an insulating material, two dissipation processes can be envisaged [59]:

(i) Surface charge spreading, which is a conservative mechanism for the charge quantity. Thus, the integrated intensity under the potential profile $\mathrm{I}_{\mathrm{s}}$ is constant with time, whereas the maximum potential $\mathrm{V}_{\mathrm{m}}$ decreases. Moreover, strong peak broadening is observed, i.e. increasing of FWHM;

(ii) Drift of charges in the bulk of the material with/without charge dissipation or recombination that appears conservative/non-conservative process for the measured potential. Indeed, when charges penetrate into the bulk layer, their influence on the tip 
decreases with the distance from the surface. The integrated intensity $I_{\mathrm{s}}$ and the maximum potential $\mathrm{V}_{\mathrm{m}}$ decrease with the same kinetics. When the two phenomena (surface diffusion and volume intake) are superimposed, the integrated intensity $I_{s}$ and the maximum potential $\mathrm{V}_{\mathrm{m}}$ decrease with different time constants. It is to note that already at the charging step, the charges spread: the lateral electric field radiated by the biased tip promotes charge lateral spreading whereas electric field at contact point controls the amount of injected charges [14].

Considering a particular application one can investigate different phenomena. The main failure mechanism in capacitive Radio Frequency MicroElectroMechanical Sytems (RF-MEMS) with electrostatic actuation is related to charging effect in the dielectric layer which induces stitching of the mobile membrane [53]. To mitigate this effect, one way is to provide dielectric layer favoring charge release, nevertheless keeping high insulating characteristics. One possibility is to create plasma processed dielectric layers with gradual variation of their electrical properties, for example amorphous silicon oxynitride layers $\mathrm{SiO}_{\mathrm{x}} \mathrm{N}_{\mathrm{y}}$ (with only 4 at.\% of $\mathrm{N}$ in the layer) [76]. In this case, the $\mathrm{Si}$ concentration is tuned in the layer by acting on the $\gamma$ parameter which reflects the ratio of the partial pressures of gas precursors in the plasma $\left(\gamma=\mathrm{N}_{2} \mathrm{O} / \mathrm{SiH}_{4}\right)$. Fig. 7 compares surface potential decay in $\mathrm{SiO}_{\mathrm{x}} \mathrm{N}_{\mathrm{y}}$ layers with different structural characteristics. Results emphasize a strong influence of the material properties on the charge decay. Indeed, in the $\mathrm{SiO}_{\mathrm{x}} \mathrm{N}_{\mathrm{y}}(\gamma=100)$ layer, which is close in composition to thermal silica, the resistivity is high $\left(22.010^{14} \Omega . \mathrm{m}\right)$ and charges remain trapped in the dielectric layer. No broadening of the potential peak and only weak $\mathrm{I}_{\mathrm{s}}$ decrease are observed. In the $\mathrm{SiO}_{\mathrm{x}} \mathrm{N}_{\mathrm{y}}(\gamma=10)$ layer, although the resistivity remains high $\left(4.910^{14} \Omega . \mathrm{m}\right)$ the charges spread laterally. The potential peak broadening is important. Moreover, an important decrease of the peak $I_{\mathrm{s}}$ area is observed. It is concluded that surface diffusion and volume intake mechanisms of charge occur simultaneously for this layer. Finally, in the $\mathrm{SiO}_{\mathrm{x}} \mathrm{N}_{\mathrm{y}}(\gamma=5)$ layer, which possesses slightly higher concentration of $\mathrm{Si}$ and consequently a bit lower resistivity $\left(3.810^{14} \Omega . \mathrm{m}\right)$ compared to $\mathrm{SiO}_{\mathrm{x}} \mathrm{N}_{\mathrm{y}}(\gamma=10)$ layer, high $\mathrm{I}_{\mathrm{s}}$ decrease is observed alongside with weak potential peak broadening. Here, the main charge release mechanism is the volume one which is ascribed to high conduction during the injection process [66].
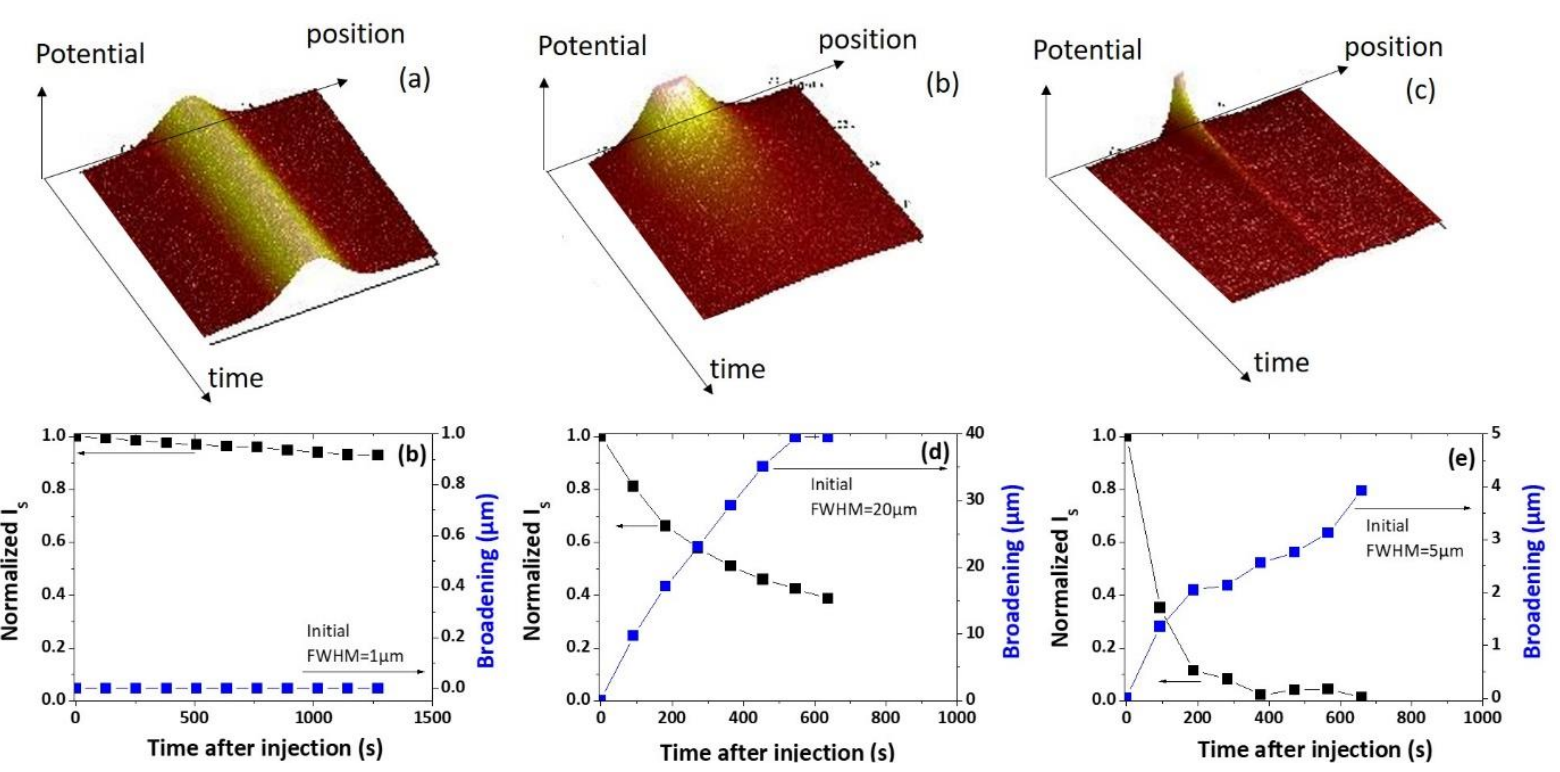

Fig. 7. Evolution of surface potential profile (a-c) and normalized $I_{s}$ and broadening (d-e) as a function of time after injection for $\mathrm{SiO}_{\mathrm{x}} \mathrm{N}_{\mathrm{y}}$ layers elaborated by plasma process with different gas ratio $\gamma$ [76]: (a,d) $\gamma=100,(b, e) \gamma=10$ and $(\mathrm{c}, \mathrm{f}) \gamma=5$. Charge injection in contact mode during $1 \mathrm{~min}$ at $25 \mathrm{~V}$.

Concerning floating gate MOS memories, a thin dielectric layer with embedded nanoparticles [77] or quantum dots appears promising for the device performance in terms of charging and retention times [78]. Lwin et al demonstrated that the work function of metallic nanoparticles has a strong influence 
on the charge decay in the device active layer [77]. Indeed, as shown on Figs. 8 the charge decay and spreading dynamics are quicker for $\mathrm{Au}$ nanoparticles than for Pt ones, with Au having lower work function compared to the Pt-work function.
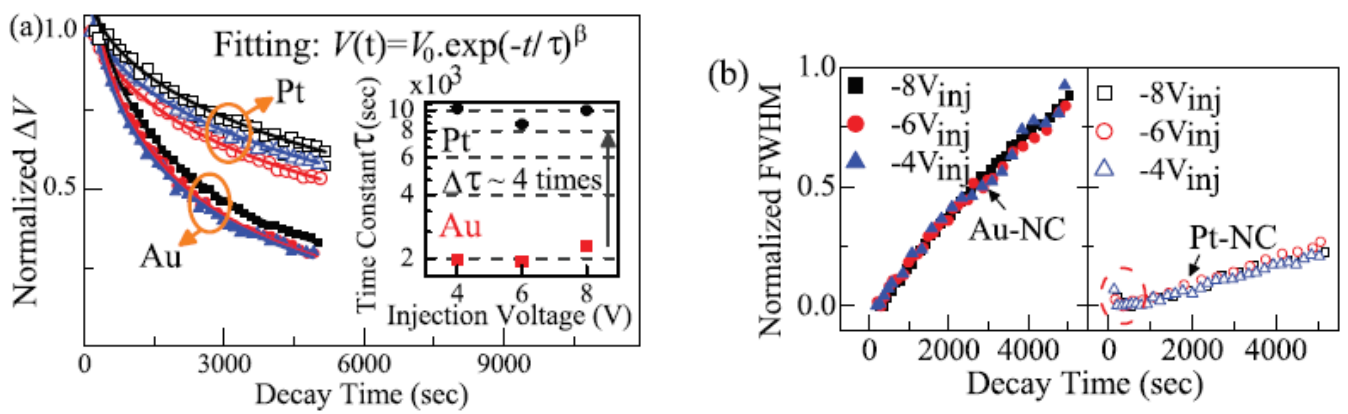

Fig. 8. Time dependence of normalized (a) maximum potential and (b) FWHM for sample with Au and $\mathrm{Pt}$ nanoparticles after charge injection of $-4 \mathrm{~V},-6 \mathrm{~V}$ and $-8 \mathrm{~V}$ during $10 \mathrm{~s}$. The inset shows characteristic time constant extracted from the curve fitting (Reproduced from Lwin et al 2011).

\subsubsection{From surface potential measurement to charge density determination}

Following the evolution of KPFM profile in time after charge injection provides information about the charge dissipation processes but to identify the physical mechanisms, actual charge density and charge distribution are necessary. Different attempts to extract charge density from KPFM measurements after local charge injection are reported in the literature. However, the two main limitations to reach this goal are related to measurements of the real surface potential and to hypotheses about the in-depth charges cloud extension. The mainly adopted hypothesis in a lot of studies is that the charges are located on the dielectric surface [63-64]. This hypothesis seems valid for charges injection using triboelectrification but appears questionable for contact/tapping mode of injection. Other hypotheses are proposed in this case like charge located at internal interface [65] or distributed in the entire volume of the dielectric layer [71].

Experimental results show that the measured surface potential is lower in intensity and broader in shape than the theoretical one mainly due to parasitic capacitance between the AFM probe and the sample. To overcome this issue, Xu et al. proposed to use PSF function (cf. part 2.2.2) to extract the real surface potential induced by the injected charge [79]. Under the hypothesis that charges are located on the surface, the authors concluded that the deconvoluted profile provides twice higher charge density with value closer to the macroscopic one.

To try to reproduce the real configuration, E. Palleau et al. investigated the influence of charges penetration in thin PMMA layers [48]. In this study charges are injected using electrostatic patterning and charge density is determined for various charges area dimensions. As shown in Table IV, for a fixed charge pattern, the charge density is influenced by hypothesis about their in-depth penetration. The more charges are considered to spread in the volume the lower is the density needed to reproduce KPFM signal.

Table IV. Charge density as function of pattern size and charge penetration depth $p$ used in the modelling [48].

\begin{tabular}{|c|c|c|c|c|}
\hline \multirow{2}{*}{ Pattern size } & \multicolumn{4}{|c|}{ Charge density $\left(\mathbf{C} / \mathbf{m}^{2}\right)$} \\
\cline { 2 - 5 } & $\mathbf{p}=\mathbf{1 n m}$ & $\mathbf{p}=\mathbf{1 0 n m}$ & $\mathbf{p}=\mathbf{5 0} \mathbf{n m}$ & $\mathbf{p}=\mathbf{1 0 0 n m}$ \\
\hline $\mathbf{1} \boldsymbol{\mu m}$ & $3.7510^{-3}$ & $3.610^{-3}$ & $2.810^{-3}$ & $1.910^{-3}$ \\
\hline $\mathbf{5} \boldsymbol{\mu m}$ & $3.6510^{-3}$ & $3.510^{-3}$ & $2.7510^{-3}$ & $1.7510^{-3}$ \\
\hline $\mathbf{1 0} \boldsymbol{\mu m}$ & $3.2510^{-3}$ & $3.0510^{-3}$ & $2.4510^{-3}$ & $1.610^{-3}$ \\
\hline
\end{tabular}

Up to now, no one of the reported methods based on KPFM measurements has permitted to extract 3D charge density without hypothesis about the charge in-depth behavior. To face this problem approaches, based on Electrostatic Force Distance Curve (EFDC) have been recently proposed [15]. 
The method based on Force Distance Curve measurements appears promising to probe space charge in 3D in thin dielectric layers due to its sensitivity to charge localization [80]. However, the EFDC modeling needs to be improved and inverse method to be developed in order to ascribe unique charge density profile to each experimental EFDC.

\section{KPFM for space charge probing in semiconductor and dielectric materials}

As shown previously, the versatility of the KPFM technique provides a guess for the fate of injected charges (lateral vs. in-depth spreading) in non-invasive way. However, sometimes the investigated structures are real devices (PN junctions, solar cells...) in which metal/dielectric, metal/semiconductor or semiconductor/semiconductor interfaces are present and should be cosidered. V. Palermo et al demonstrated that the KPFM is a powerful technique to characterize thin films for electronic applications, however without extracting information about the amount of related charge [26]. This points to the need of methodology for estimation of the charge distributions using KPFM to investigate charge generated in processes under external stress (electric field, light...) In the following, methodologies developed to extract charge density profile from surface potential measurement are presented, before addressing their application to different devices.

\subsection{KPFM measurements on bias electronic devices: challenge and bottleneck}

During KPFM measurements over real biased electronic devices (organic/inorganic FET, CMOS, solar cells...), the bias applied on electrodes (Fig. 9) induces electrostatic forces which impact the measured topography. The apparent height effect discussed above decreases the KPFM sensitivity close to the electrodes [81]. A way to avoid this effect is to investigate charge behavior after bias application. This method is mainly valid for dielectric layers due to charge trapping effect. Another way is to exploit the Feed-forward compensation proposed by Ziegler et al during KPFM measurements [72]. More recently, Bercu et al proposed a modified AM-KPFM set-up which avoids error on surface topography due to biased electrodes [82]. The proposed system uses an external voltage source (for electrode biasing) and synchronized AFM tip which permits to apply bias on the electrode only during surface potential measurements.
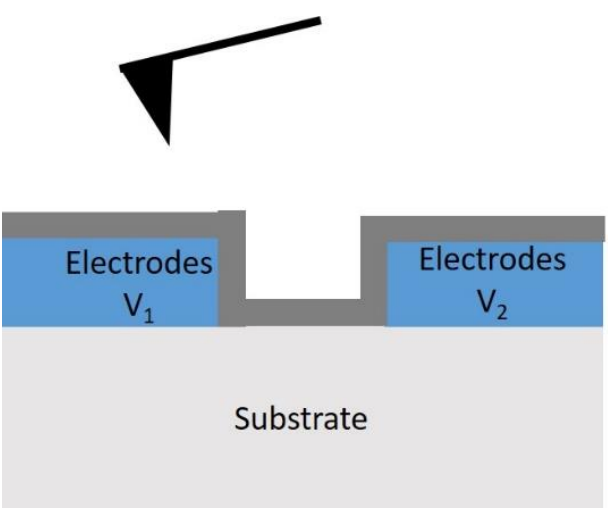

Fig. 9. KPFM configuration during measurement over real device under bias. In grey, dielectric or semiconductor active probed layer.

\subsection{Methodology for charge density profile determination from KPFM measurements}

To extract the space charge density profile $\rho(\mathrm{x})$ from the surface potential profile $\mathrm{V}_{\mathrm{s}}(\mathrm{x})$ measured by KPFM three approaches were explored in the literature based on solution of the Poisson's equation for electrostatics. The first approach, named Second Derivative Model (SDM) is the simplest one and relies on the hypothesis that the probed surface potential is representative of the potential distribution in the layer volume. Here, the charge distribution is deduced from the second derivative of the measured surface potential ( $\varepsilon_{0}$ is the vacuum permittivity, $\varepsilon_{\mathrm{r}}$ the relative permittivity of the dielectric material): 


$$
\rho(x)=-\varepsilon_{0} \varepsilon_{r} \frac{d^{2} V_{S}}{d x^{2}}
$$

The spatial resolution in this approach is sensitive to the derivation step $d x$ and to the noise level superimposed to the KPFM recorded profile [83]. Refining the derivation step is mandatory to improve spatial resolution. This method is also used, in two dimensions, to extract charge density profile for localized injected charge [84].

The second approache is based on the same hypothesis (charge distribution is obtained using eq. 11), but prior to the derivation step a signal treatment is applied on the raw data to reduce noise impact [85]. The most powerful smoothing method for derivation approach is Savitzky and Golay (SG) one [86]. In this method a fixed degree polynomial function is used to fit the experimental data on a fixed amount of equally-spaced data points named "data point window". The fitting process is based on least squares polynomial regression. In the follow this method will be named SG-SDM. Using this method Faliya et al optimized the technique in term of "data point window" and noise robustness [85].

The last method is based on Finite Element Model (FEM). The main advantage of this method is to reproduce the real sample geometry without assumption on the potential distribution in 3D [87]. The Poisson equation is solved in two dimensions in the dielectric layer and in the surrounding air box to determine the potential distribution $\mathrm{V}(\mathrm{x}, \mathrm{z})$ :

$$
\frac{d^{2} V}{d x^{2}}+\frac{d^{2} V}{d z^{2}}=\frac{\rho(x, z)}{\varepsilon_{0} \varepsilon_{r}} .
$$

In this model, a hypothesis on the shape of the charge density cloud is needed. The process followed to determine charge density is a sequential one: (i) Initial charge density profile is supposed; (ii) The surface potential map is computed using eq. 12 and compared to the experimental one; (iii) If the difference is less than the KPFM resolution the charge density is determined, otherwise the initial charge density is modified a

nd the surface potential is computed till convergence with experimental profile.

\subsection{Applications to dielectrics and semiconductors}

\subsubsection{Charge dynamic in solid electrolytes}

The development of improved lithium-ion batteries needs understanding of the charge dynamic inside the solid polymer electrolyte. In this perspective, KPFM measurements appear very appropriate. In the last decade numerous studies focused on the charge dynamic in poly(ethylene oxide) (PEO), a ion conductor polymer, which is promising material for solid electrolyte applications. In this context, Martin et al extracted charge density profile in PEO using the SDM method. However, due to poor signal/noise ratio the obtained results are not subject to interpretation [88]. Few years later, Faliya et al. proposed the SG-SDM method to overcome the influence of noise on the surface potential measurements [85]. Optimizing the SG smoothing process, the authors managed to extract space charge density in PEO (Fig. 10.c) from noisy surface potential profiles (Fig. 10.a). Following the same approach results were obtained for the PEO layer after applying external stress and charge oscillation in time (Figs. 10. b and d). Note that in this last case, the influence of the electric field on topography is not compensated. 

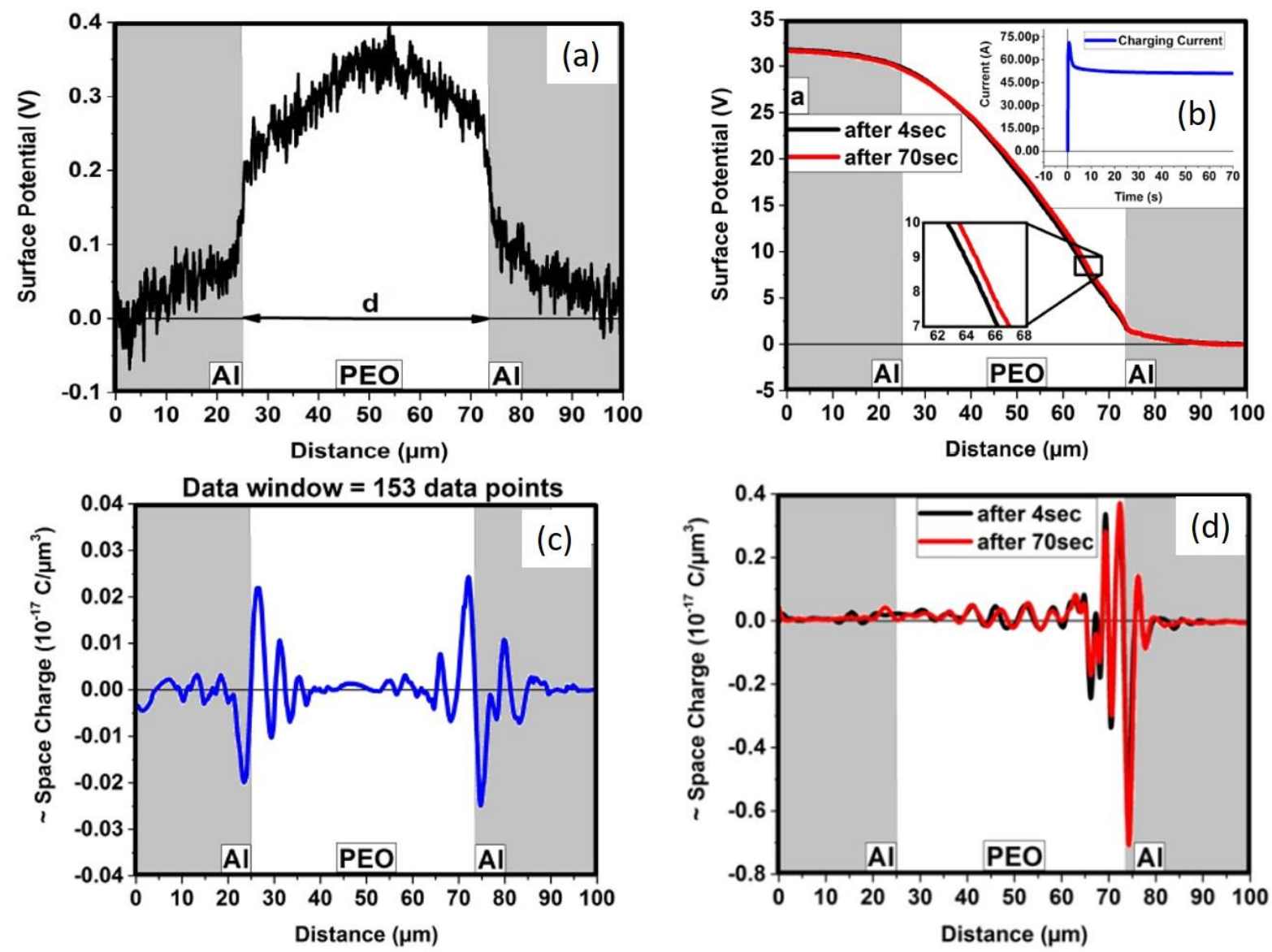

Fig. 10. Surface potential profile measured by KPFM over Al/PEO/Al structure (a) without applied bias and (b) 30V (ground) applied on left (right) electrode. Resulting space charge density profile obtained using SG-SDM method (c) without applied bias and (d) 30V (ground) applied on left (right) electrode. (Reproduced from Faliya et al [84])

\subsubsection{Charge density in thin-films transistors}

KPFM was used to investigate charge density distribution in thin-films transistors. Due to mobile charges, which are present and transported continuously in the device, the KPFM measurements and interpretation of the obtained profiles in operating transistor devices appear challenging [25]. In this case the surface potential is measured by KPFM between source and drain with or without applied potential. The resulting surface potential profile is being interpreted using the SDM method to obtain two kinds of information. The first one is related to the investigation of different doped regions. Applying this methodology to a doped drain of a metal-oxide-silicon field effect transistor, Henning et al distinguished relative changes in dopant concentration with lateral resolution of less than $100 \mathrm{~nm}$ [89]. They emphasize that, even though this method does not provide absolute dopant concentration, it is sensitive to changes in the dopant concentration, from $10^{15}$ to $10^{20} \mathrm{~cm}^{-3}$. The same kind of information was obtained by Kryvchenko et al applying bias between drain and source to accentuate the contrast in surface potential and to improve resolution (Figs. 11) [90]. However, as shown on Fig. 11.b these authors do not reach quantitative values for the amount of charge. The second kind of information concerns process issues. As an example, Kryvchenko et al investigated contact metal diffusion in $\operatorname{In}_{2} \mathrm{O}_{3}$ thin-film transistor devices [90]. As shown on Fig. 11.a, surface potential close to the right electrode is poorly reproduced by Technology Computer-Aided Design (TCAD) software even without applied voltage on the drain. Indeed, charges are located on this electrode (Fig.11.b) which is due to contact metal diffusion. 
(a)

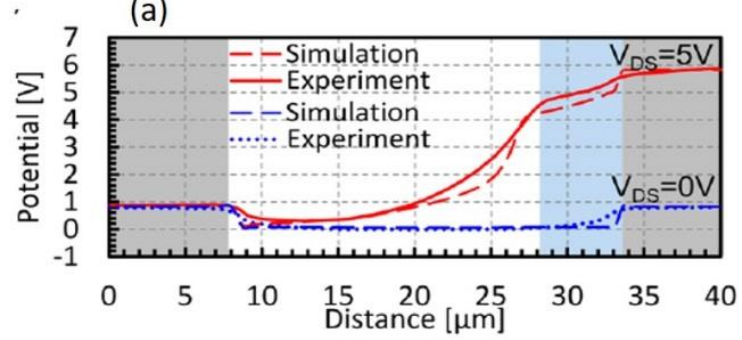

(b)

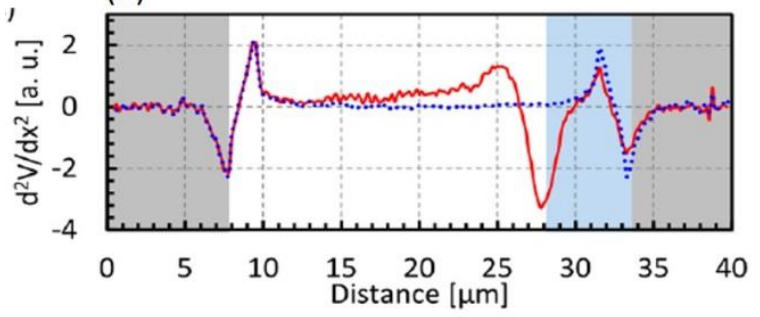

Fig. 11. (a) Comparison of surface potential profile measured by KPFM and simulated by TCAD software at $\mathrm{V}_{\mathrm{DS}}=0 \mathrm{~V}$ and $\mathrm{V}_{\mathrm{DS}}=5 \mathrm{~V}$ when $\mathrm{V}_{\mathrm{GS}}=0 \mathrm{~V}$. (b) Calculated second derivative of the measured surface potential at $\mathrm{V}_{\mathrm{DS}}=0 \mathrm{~V}$ (dashed line) and $\mathrm{V}_{\mathrm{DS}}=5 \mathrm{~V}$ (solid line) represent the measured charge profiles (Reproduced with permission from Kryvchenko et al [90]. Copyright (2016) American Chemical Society)

\subsubsection{Injection at metal dielectric interface}

KPFM measurements were used to investigate charge injection at metal/dielectric interface. As an example Okamoto et al investigated the influence of $\mathrm{BaTiO}_{3}$ degradation close to the anode for multilayer ceramic capacitor applications [91]. In another study, the charge injection at $\mathrm{SiN}_{\mathrm{x}} / \mathrm{Al}$ interface was investigated using FM-KPFM measurements after lateral Al-electrode polarization. Fig. 12.a represents the resulting surface potential with negative peak close to cathode and positive peak to anode. Due to the profile shape, a Gaussian distribution of the charges density at both electrodes is supposed. Thus, for positive and for negative charges, the density is expressed as:

$$
\rho(x)=\rho_{0} \exp \left(-\frac{\left(x-x_{0}\right)^{2}}{0.36 W^{2}}\right)
$$

where $\rho_{0}$ is the maximum value (positive for holes and negative for electrons), $x_{0}$ is the position at which the maximum density occurs and W is the FWHM of the charges cloud.

SDM and FEM methods were applied to the FM-KPFM profile showing that both methods provide the the same shape for charge density (Fig. 11.b). However, the amount of charges found is different [87]. Indeed, the authors demonstrate that the FEM method, which reproduces the real geometry of the device, provides more representative value for the charge density. Therefore, for quantitative charge density determination the FEM method should be preferred.

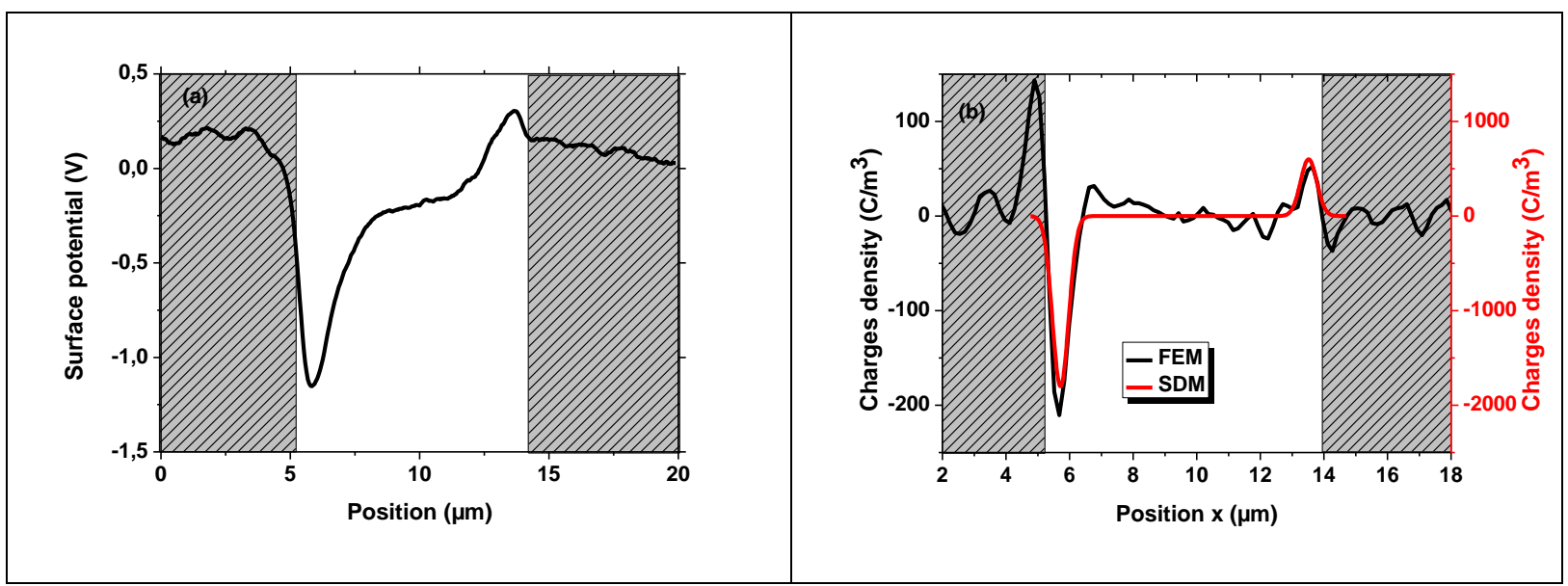

Fig. 12 (a) Surface potential profile probed by FM-KPFM and (b) charge density profiles extracted from the surface potential profile measured using the SDM and the FEM models. 


\section{Nanoscale opto-electrical characterization of thin film based solar cells using KPFM and C-AFM}

Energy production is the defining challenge of the 21 st century as the global demand is projected to more than double by 2050 [92]. An exponential increase of the production capacity from the solar energy is highly required. Two approaches have been proposed to achieve cheaper photovoltaic (PV) electricity [11]:

(i) Increasing the power conversion efficiency while keeping PV-materials costs the same (Si-wafer-based solar cells (1st generation) or high efficiency concepts ("all-Si" tandem cells for 3rd generation);

(ii) Developing low-cost, moderate efficiency PV-materials (thin-film PV, 2nd generation). Organic photovoltaic (OPV) are an important emerging technology, part of 2nd generation, which promise to provide solar-to-electric energy conversion in portable, light-weight packages, at extremely low cost;

Independently on the technology, PV or OPV, device performances are controlled by phenomena occurring at nanoscale (exciton formation by light, excitation dissociation at $\mathrm{p} / \mathrm{n}$ junction, carriers transport in $\mathrm{p}$ or $\mathrm{n}$ layers/domains). So, charges creation under illumination and their transport in the active layer should be investigated at local scale. To that end, as shown in the following, three kinds of measurements are implemented:

(i) Current or surface potential mapping with or without illumination to localize the area where charges are created or collected by top/bottom electrode;

(ii) Localized current versus voltage measurements to investigate charge transport mechanisms and determine material properties as charge mobility;

(iii) Time resolved measurements to follow the current or surface potential evolution in time during light/dark transition and to investigate charge dynamics;

These techniques first developed for solar cells are now frequently applied for photoelectrochemical cells involved in water splitting applications [93].

\subsection{Mapping measurements}

The influence of illumination on surface potential [94-96] or current [97-100] maps was extensively studied in the literature. Figs. 13.a and 13.b show the evolution of surface potential map over PFB:F8BT blend film for different light wavelengths. At long wavelengths, light does not create excitons in the both materials due to the low absorption (Fig. 13.a) and surface potential is not modified. According to results from absorbance spectroscopy, other wavelengths are chosen to excite only F8BT $(370 \mathrm{~nm})$ or for both materials inducing a decrease of their surface potential due to the photocharge effect. 


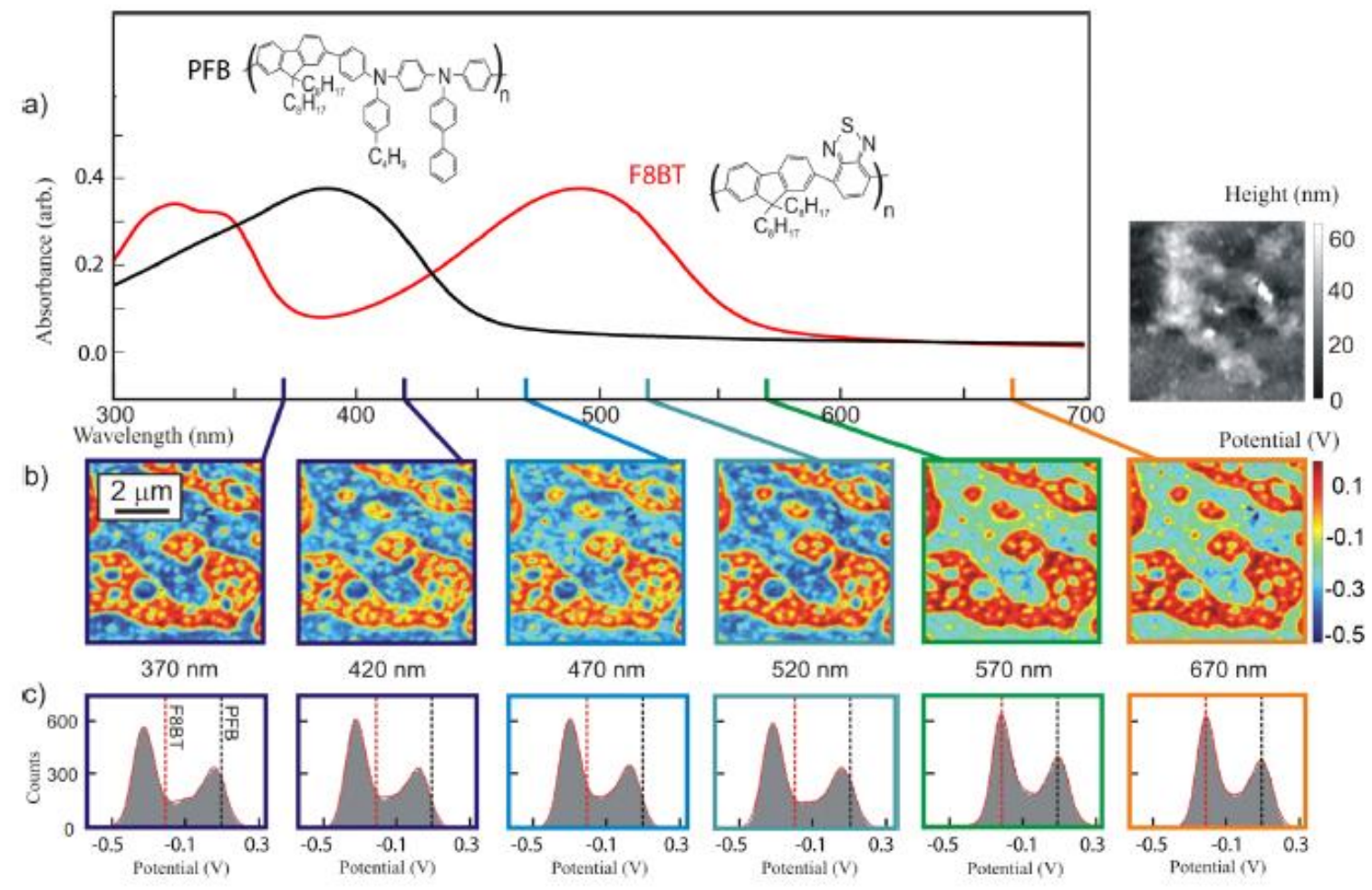

Fig. 13. Evolution of surface potential map of PFB:F8BT film illuminated under different wavelengths (Reprinted with permission from J. Luria [94]. Copyright 2012 American Chemical Society.")

\subsection{Localized current-voltage measurements}

Concerning electrical measurements at local scale two kinds of measurements can be performed: current versus voltage and time resolved characterization. According to the literature, current versus voltage measurements are the most frequently performed ones. Direct interpretation of the obtained results provides information for the voltage threshold evolution as a function of light power [93] or for the current as a function of light wavelength or for minimum photon energy sufficient for the photoexcitation of mobile charge carriers [101]. However, deeper analysis of the results is not straightforward. The main limits are imposed by the used model and determination of the collection surface. Indeed, the electrical characteristics were mainly obtained on the basis of Space Charge Limited Current (SCLC) model to extract carrier mobilities [102]. However, many studies point out that the mobility determined using C-AFM measurements is of some orders of magnitude higher than the mobility obtained in macroscale way [103]. This difference is related to the tip-plane configuration during C-AFM measurements. Indeed, this configuration induces inhomogeneous electric field distribution with large field enhancement close to the tip compared to the one computed in plane-plane configuration. Reid et al proposed a modified SCLC model to consider the actual electric field (and current density inhomogeneity) due to the tip-plane configuration [103]. This approach provides carrier mobility in the same order of magnitude as the C-AFM and macroscale measurements.

Concerning determination of the collection surface some improvements have been proposed:

(i) Contact area is computed using the Hertz approach and corresponds to the mechanical contact area [104];

(ii) Effective surface is determined by fitting current-voltage experimental curve. This surface depends on several parameters like tip work function, contact force and dielectric thickness, and presents a broad range of values from $10 \mathrm{~nm}^{2}$ to $100 \mathrm{~nm}^{2}$ [105];

(iii) SEM observations of the AFM tip after measurements [40]. Moeman et al proposed an approach to estimate the radius of area in which the current is collected as function of the sample mechanical properties [106]. 
Time resolved electrical measurements consist in acquiring surface potential and / or current in time during or after illumination to probe the charge transport. Figs. 14 shows surface potential and current evolution during light and dark cycle. After illumination surface potential decrease whereas current increase. The response time can be related to the device performances. Indeed, D. Coffey reported that the response time is determined by the solar cell external quantum efficiency [107].
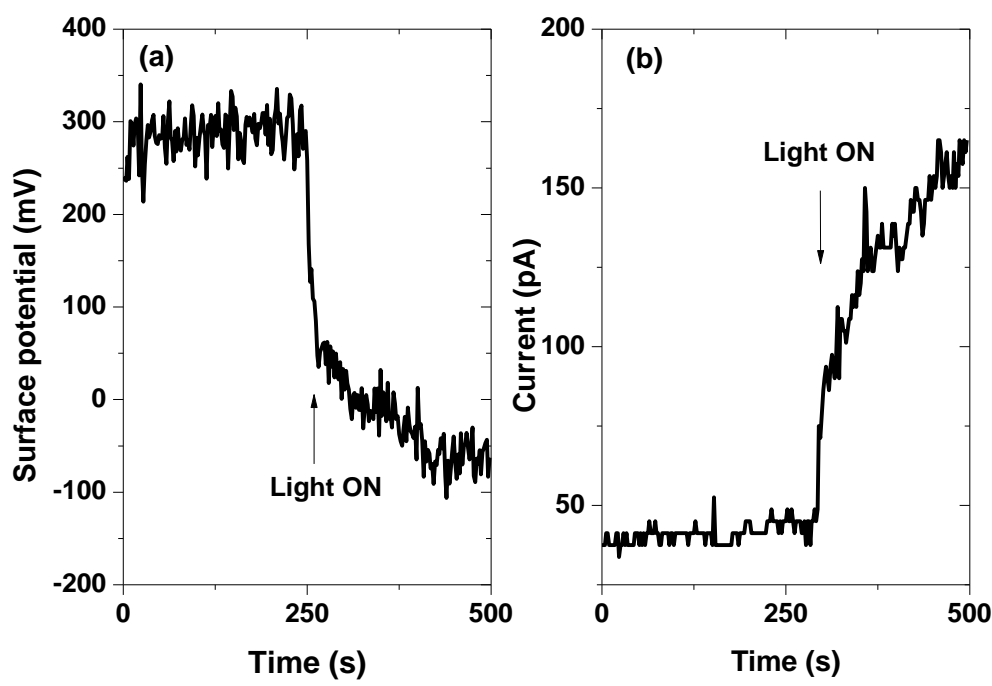

Fig. 14. Evolution of (a) photovoltage and (b) photo-current (polarization bias of $3 \mathrm{~V}$ ) for 40nm-thick P3HT:PCBM blend.

Current versus voltage and time resolved measurements provide complementary information on the electrical properties of devices. Indeed, combining these techniques C.Villeneuve-Faure et al showed that in the P3HT:PCBM blend charges are trapped during light exposition which induces a decrease of the Schottky barrier to injection and a modification of the current versus voltage characteristics [108].

\section{Conclusion and overview}

KPFM and C-AFM appear as powerful techniques to investigate physical phenomena related to charges injection and transport in the active layers of different devices. However, their performances remain limited by the instrumental set-up achievements and existing methodologies for interpretation of the obtained results. From experimental point of view the KPFM and C-AFM performances are mainly limited by the tip characteristics. Indeed, the AFM tips used for electrical measurements are silicon-tips with conductive coating and present greater tip radius than silicon one (from $25 \mathrm{~nm}(\mathrm{Pt}$ coating) to $125 \mathrm{~nm}$ (diamond-coating) versus only around $5 \mathrm{~nm}$ for bare Si-tip). This implies worse spatial resolution either in topography or in electrical properties and higher parasitic capacitance (lower sensitivity). To improve performance new probes are under development as Si-doped conductive probes used for PF-KPFM or PF-TUNA modes.

The results interpretation appears strongly limited by the lack of appropriate modelling of the interaction between the AFM probe and the studied sample (electrostatic force, contact area...). Concerning space charge measurements by using KPFM, 2D-measurement methods are available and can be used to investigate charge injection and transport at the interfaces appearing in many devices. However, up to now no existing technique allows to probe localized charges in 3D without hypothesis on the charge distribution (shape, in-depth penetration...). This issues need mutual evolution of both the AFM instrument with new probing method really sensitive to the charge localization in the volume and the modelling of electrostatic interaction between the AFM tip and the sample (real tip geometry as example). Concerning charge dynamic probing by C-AFM, the drawbacks are mainly related to 
results interpretation. The used modelling approaches should be improved to consider the real sample geometry (and the related electric field heterogeneity) and the collection surface to extract the electrical properties of the studied devices.

\section{References}

[1] A. Galembeck, C.A.R. Costa, M.C.V.M. da Silva, E.F. Souza, F. Galembeck, "Scanning electric potential microscopy imaging of polymers: electrical charge distribution in dielectrics" Polymer 42, $4845(2001)$

[2] D.J. Lacks and R. M. Sankaran, "Contact electrification of insulating materials" J. Phys. D: Appl.d Phys. 44, 453001 (2011)

[3] T. Shinbrot, M. Rutala and H. Herrmann "Surface contact charging" Phys. Rev. E 96, 032912 (2017)

[4] G. Bersuker, P. Zeitzoff, G. Brown, H.R. Huff, " Dielectrics for future transistors", Materials Today 7, 26-33 (2004)

[5] D.J. Fonseca, M. Sequera, "On MEMS reliability and failure mechanisms" International Journal of Quality, Statistics, and Reliability 2011, 820243 (2011)

[6] B. Bhushan, "Nanotribology and nanomechanics of MEMS/NEMS and BioMEMS/BioNEMS materials and devices" J. Microelectronic Engineering 84, 387 (2007)

[7] U. Zaghloul, G. Papaioannou, B. Bhushan, F. Coccetti, P. Pons, R. Plana, "On the reliability of electrostatic NEMS/MEMS devices: review of present knowledge on the dielectric charging and stiction failure mechanisms and novel characterization methodologies", Microelectronics Reliability 51, 1810-1818 (2011).

[8] U. Zaghloul, B. Bushan, P. Pons, G. J. Papaioannou, F. Coccetti, and R. Plana, "On the influence of environment gases, relative humidity and gas purification on dielectric charging/discharging processes in electrostatically driven MEMS/NEMS devices," Nanotechnology 22, 035705 (2011).

[9] W. Zhou, J.B. He, X.P. He, H.J. Yu, B. Peng, "Dielectric charging induced drift in micro device reliability-a review", Microelectronics Reliability 66, 1-9 (2016).

[10] C. Zhao, C. Zhou Zhao, S. Taylor and P.R. Chalker, "Review on non-volatile memory with high$\mathrm{k}$ dielectrics:Flash for generation beyond $32 \mathrm{~nm}$ ", Materials 7, 5117-5145 (2014).

[11] M.C. Scharber, N.S. Sariciftci "Efficiency of bulk-heterojunction organic solar cells" Progress in Polymer Science, 38 1929-1940 (2013)

[12] J. Le Rouzo, D. Duché, C. Ruiz, F. Thierry, M. Carlberg, G. Berginc, M. Pasquinelli, J.J. Simon, L. Escoubas, F. R. Flory "Specific tools for studying the optical response of heterogeneous thin film layers" J. Nanophotonics 11, 016009 (2017)

[13] M.Y. Li, C.H. Chen, Y. Shi and L.J. Li "Heterostructures based on two-dimensional layered materials and their potential applications" Materials Today 19, 322-335 (2016)

[14] C. Villeneuve-Faure, K. Makasheva, L. Boudou, G. Teyssedre, "Charge injection in thin dielectric films by Atomic Force Microscopy: Influence of geometry and material work function of the AFM tip on the injection process", Nanotechnology 27, 245702 (2016)

[15] C. Villeneuve-Faure, L. Boudou, K. Makasheva and G. Teyssedre "Atomic Force Microscopy Developments for Probing Space Charge at Sub-micrometer Scale in Thin Dielectric Films" IEEE Transactions on Dielectrics and Electrical Insulation 23, 713 (2016) 
[16] S. Diaham, F. Saysouk, M.-L. Locatelli and T. Lebey, "Huge nanodielectric effects in polyimide/boron nitride nanocomposites revealed by the nanofiller size" J. Phys. D: Appl. Phys. 48, 385301 (2015)

[17] T. Takada, Y. Hayase, Y. Tanaka and T. Okamoto "Space charge trapping in electrical potential well caused by permanent and induced dipoles for LDPE/MgO nanocomposite" IEEE Trans. Dielectr. Electr. Insul. 15, 152-160 (2008)

[18] G. Teyssedre, S.T. Li, K. Makasheva, N. Zhao, L. Milliere and C. Laurent "Interface tailoring for charge injection control in polyethylene" IEEE Trans. Dielectr. Electr. 24, 1319-1330 (2017)

[19] P. Van Rysselberghe, "A Note on Work Functions and Chemical Potentials" J. Chem. Phys. 21, 1550 (1953)

[20] M. Nonnenmacher, M.P. O’Boyle, H.K. Wickramasinghe "Kelvin probe force microscopy" Appl. Phys. Lett. 58, 2921 (1991)

[21] Y. Rosenwaks, R. Shikler, T. Glatzel and S. Sadewasser "Kelvin probe force microscopy of semiconductor surface defects” Phys. Rev. B 70, 085320 (2004)

[22] Y. Shen, D.M. Barnett and P.M. Pinsky "Simulating and interpreting Kelvin probe force microscopy images on dielectrics with boundary integral equations" Rev. Sci. Instrum. 79, 023711 (2008)

[23] R. Shikler, T. Meoded, N. Fried, B. Mishori, Y. Rosenwaks "Two-dimensional surface band structure of operating light emitting devices” J. Appl. Phys. 86, 107 (1999)

[24] H.O. Jacobs, P. Leuchtmann, O.J. Homan and A. Stemmer "Resolution and contrast in Kelvin probe Force microscopy" J. Appl. Phys. 84, 1168 (1998).

[25] A. Liscio, V. Palermo, K. Mullen and P. Samori "Tip-sample interaction in Kelvin Probe Force Microscopy: Quantitative measurement of the local surface potential" J. Phys. Chem. C 112, 17368 (2008)

[26] V. Palermo, M. Palma and P. Samori "Electronic Characterization of Organic Thin Films by Kelvin Probe Force Microscopy" Adv. Matter 18, 145 (2006)

[27] U. Zerweck, C. Loppacher, T. Otto, S. Grafström and L.M. Eng "Accuracy and resolution limits of Kelvin probe force microscopy" Phys. Rev. B 71, 125424 (2005)

[28] W. Meliz, J Shen, A. C. Kummel and S. Lee "Kelvin probe force microscopy and its application" Surface Science Reports 66, 1 (2011)

[29] M. Bieletzki, T. Hynninen, T. Soini, M. Pivetta, C. Henry, A. Foster, F. Esch, C. Barth and U. Heiz "Topography and work function measurements of thin $\mathrm{MgO}(001)$ films on $\mathrm{Ag}(001)$ by nc-AFM and KPFM" Phys. Chem. Chem. Phys. 12, 3203 (2010)

[30] C. Barth and C.R. Henry "Kelvin probe force microscopy on surfaces of UHV cleaved ionic crystals" Nanotechnology 17, 5155 (2006)

[31] E. Strassburg, A. Boag and Y. Rosenwaks "Reconstruction of electrostatic force microscopy images" Rev. Sci. instrum. 76, 083705 (2005)

[32] G. Cohen, E. Halpern, S.U. Nanayakkara, J.M. Luther, C. Held, R. Bennewitz, A. Boag and Y. Rosenwaks "Reconstruction of surface potential from Kelvin probe force microscopy images" Nanotechnology 24, 295702 (2013)

[33] S. Sadewasser, C. Leendertz, F. Streicher and M. C. Lux-Steiner "The influence of surface topography on Kelvin Probe Force Microscopy" Nanotechnology 20, 505503 (2009) 
[34] M. Lee, M. Lee and F. B. Prinz "Geometric artefact suppressed surface potential measurement" Nanotechnology 17, 3728 (2006)

[35] D. Ziegler and A. Stemmer "Force gradient sensitive detection in lift-mode Kelvin Probe Force microscopy" Nanotechnology 22, 075501 (2011)

[36] G. Li, B. Mao, F. Lan, and L. Liu "Practical aspects of single-pass scan Kelvin probe force microscopy” Rev. Sci. instrum. 83, 113701 (2012)

[37] C. Li, S. Minne, Y. Hu, J. Ma, J. He, H. Mittel, V. Kelly, N. Erina, S. Guo and T. Mueller "PeakForce Kelvin Probe Force Microscopy" Bruker Application Note \#140 (2013)

[38] B. Pittenger, N. Erina and C. Su "Quantitative Mechanical Property Mapping at the Nanoscale with PeakForce QNM” Bruker Application Note \#128 (2012)

[39] P. Eyben, M. Xu, N. Duhayon, T. Clarysse, S. Callewaert, W. Vandervorst "Scanning spreading resistance microscopy and spectroscopy for routine and quantitative two-dimensional carrier profiling" J. Vac. Sci. Technol. B 20, 471 (2002)

[40] V. Yanev, T. Erlbacher, M. Rommel, A.J. Bauer and L. Frey «Comparative study between conventionnal macroscopic I-V techniques and advanced AFM based methods for electrical characterization of dielectrics at nanoscale » Microelectronic Engineering 86, 1911 (2009).

[41] C. Li, S. Minne, B. Pittenger, A. Mednick, M. Guide and T.-Q. Nguyen "Simultaneous Electrical and Mechanical Property Mapping at the Nanoscale with PeakForce TUNA" Bruker Application Note \#132 (2011)

[42] U. Celano "Metrology and Physical Mechanisms in New Generation Ionic Devices" Springer thesis (2016).

[43] R. Giridharagopal, G. Shao, C. Groves and D. S. Ginger1 "New SPM techniques for analyzing OPV materials" Materials Todays 13, 50 (2010)

[44] D. E. Sviridov and V. I. Kozlovsky "Toward reliable photoconductive atomic force microscopy measurements" J. Vac. Sci. \& Technol. B 34, 061801 (2016)

[45] C. Riedel, A. Legria, R. Arinero, J. Colmenero and J.J. Saenz "Contrast inversion in electrostatic force microscopy imaging of trapped charges: tip-sample distance and dielectric constant dependence" Nanotechnology 22, 345702 (2011)

[46] M. Labardi, J. Barsotti, D. Prevosto, S. Capaccioli, C.M. Roland and R. Casalini "Extended model for the interaction of dielectric thin films with an electrostatic force microscope probe" J. Appl. Phys. 118, 224104 (2015)

[47] R. Arinero, C. Riedel and C. Guasch "Numerical simulations of electrostatic interactions between an atomic force microscopy tip and a dielectric sample in buried nano-particules" J. Appl. Phys. 112, $114313(2012)$

[48] E. Palleau, L. Ressier, L. Borowik and T. Melin "Numerical simulations for a quantitative analysis of AFM electrostatic nanopatterning on PMMA by Kelvin Force Microscopy" Nanotech. 21, $225706(2010)$

[49] A. Boularas, F. Baudoin, G. Teyssedre, C. Villeneuve-Faure and S. Clain "3D modelling of electrostatic interaction between AFM probe and dielectric surface: Impact of tip shape and cantilever contribution" IEEE Transactions on Dielectrics and Electrical Insulation 23, 705 (2016)

[50] D.S.H. Charrier, M. Kemerink, B. E. Smalbrugge, T. de Vries and R. A. J. Janssen "Real versus Measured Surface Potentials in Scanning Kelvin Probe Microscopy" ACSNano 2, 622 (2008)

[51] L. Borowik, K. Kushiaku, D. Theron and T. Melin "Calculating Kelvin force microscopy signals from static force field” Appl. Phys. Lett. 96, 103119 (2010) 
[52] P. Normand, E. Kapetanakis , P. Dimitrakis , D. Skarlatos, D. Tsoukalas , K. Beltsios , A. Claverie, G. Benassayag, C. Bonafos, M. Carrada, N. Cherkashin, V. Soncini, A. Agarwal, Ch. Sohl , M. Amee "Effects of annealing conditions on charge storage of Si nanocrystal memory devices obtained by low-energy ion beam synthesis," Microelectron. Eng. 67-68, 629 (2003)

[53] A. Witvrouw, H.A.C. Tilmans and I. De Wolf I "Materials issues in the processing, the operation and the reliability of MEMS" Microelectron. Eng. 76, 245 (2004)

[54] A. Kerber, E. Cartier, R. Degraeve, P.J. Roussel, L. Pantisano, T. Kauerauf, G. Groeseneken, H.E. Maes and U. Schwalke "Charge trapping and dielectric reliability of $\mathrm{SiO} 2-\mathrm{A} 12 \mathrm{O} 3$ Gate stacks with TiN electrodes" IEEE Trans. Electron Dev. 50, 1261 (2003)

[55] J.E. Stern, B.D. Terris, H.J. Mamin and D. Rugar "Exposition and imaging of localized charge on insulator surfaces using force microscope" Appl. Phys. Lett. 53, 2717 (1988)

[56] F. Marchi, R. Dianoux, H.J.H. Smilde, P. Mur, F. Comin and J. Chevrier "Characterisation of trapped electric charge carriers behaviour at nanometer scale by electrostatic force microscopy" J. Electrostatics 6, 538 (2008)

[57] J. Lambert, G. de Loubens, C. Guthmann and M. Saint-Jean M "Dispersive charge transport along the surface of an insulating layer observed by electrostatic force microscopy" Phys. Rev. B 71, 155418 (2005)

[58] C.A. Rezende, R.F. Gouveia, M.A. da Silva and F. Galembeck "Detection of charge distribution in insulator surfaces" J. Phys.: Condens. Matter 21, 263002 (2009)

[59] S. Morita and Y. Sugawara "Microscopic contact charging and dissipation" Thin Solid Films 393, $310(2001)$

[60] R. Williams and M. H. Woods "High electric fields in silicon dioxide produced by corona charging” J. Appl. Phys. 44, 1026 (1973)

[61] I.D. Baikie, S. Mackenzie, P.J.Z. Estrup, J.A. Meyer "Noise and the Kelvin method" Rev. Sci. Instrum. 62, 1326 (1991)

[62] C. Schonenberger "Charge Sow during metal-insulator contact" Phys. Rev B 45, 3861 (1992)

[63] N. Balke, P. Maksymovych, S. Jesse, I. I. Kravchenko, Q. Li and S. V. Kalinin "Exploring Local Electrostatic Effects with Scanning Probe Microscopy: Implications for Piezoresponse Force Microscopy and Triboelectricity" ACS Nano 8, 10229 (2014)

[64] Y. S. Zhou, S. Wang, Y. Yang, G. Zhu, S. Niu, Z.-H. Lin, Y. Liu and Z. L. Wang « Manipulating Nanoscale Contact Electrification by an Applied Electric Field» Nano Lett. 14, 1567 (2014)

[65] Y. Han, Z. Huo, X. Li, G. Chen, X.Yang, D. Zhang, Y. Wang, T. Ye, and M. Liu "Investigation of Charge Loss Mechanism of Thickness-Scalable Trapping Layer by Variable Temperature Kelvin Probe Force Microscopy” IEEE Electron Devices Letters 34, 870 (2013)

[66] C. Villeneuve-Faure, K. Makasheva, C. Bonafos, B. Despax, L. Boudou, P. Pons, and G. Teyssedre « Kelvin force microscopy characterization of charging effect in thin a-SiOxNy:H layers deposited in pulsed plasma enhanced chemical vapor deposition process by tuning the Siliconenvironment" J. Appl. Phys. 113, 204102 (2013)

[67] R Diaz, E Palleau, D Poirot , N M Sangeetha and L Ressier "High-throughput fabrication of anticounterfeiting colloid-based photoluminescent microtags using electrical nanoimprint lithography" Nanotechnology 25, 345302 (2014)

[68] M. Saint Jean, S. Hudlet, C. Guthmann, and J. Berger "Charge dynamics and time evolution of contact potential studied by atomic force microscopy" Phys. Rev. B 56, 15391 (1997) 
[69] M. Chiesa and R. Garcia «Nanoscale space charge generation in local oxidation nanolithography" Appl. Phys. Lett. 96, 263112 (2010)

[70] L Ressier and V Le Nader "Electrostatic nanopatterning of PMMA by AFM charge writing for directed nano-assembly" Nanotechnology 19, 135301 (2008)

[71] K. Sridhara, Ł. Borowik, D. Mariolle, N. Chevalier and J.-P. Colonna "Environment and baking influence on charge retention on silicon nitride charge trap layers" J. Appl. Phys. 111, 023710 (2012)

[72] D. Ziegler, N. Naujoks, and A. Stemmer «Feed-forward compensation of surface potential in atomic force Microscopy » Rev. Sci. Instrum. 79, 063704 (2008)

[73] S. Morita, Y. Sugawara and Y Fukano "Atomic force microscope combined with scanning tunneling microscope” Japan. J. Appl. Phys. 32, 2983 (1993)

[74] K. Makasheva, C. Villeneuve-Faure, C. Laurent, B. Despax, L. Boudou and G. Teyssedre "Dielectric charging by AFM in tip-to-sample space mode: overview and challenges in revealing the appropriate mechanisms" Nanotechnology 26, 295704 (2015)

[75] Z. Sun, X. Wang, W. Song and Q. Lei "A research on the surface charge with different coating tip by electrostatic force microscope" Annu. Rep. IEEE Conf. Electrical Insulation Dielectric Phenomena 295 (2013)

[76] K. Makasheva, B. Despax, L. Boudou, and G. Teyssedre, "Dielectric layers for RF-MEMS switches: design and study of appropriate structures preventing electrostatic charging," IEEE Trans. Dielectrics and Electrical Insulation, 19, 1195 (2012)

[77] Z. Z. Lwin, K. L. Pey, C. Liu, Q. Liu, Q. Zhang, Y. N. K. Singh and S. Mahapatra "Localized charge trapping and lateral charge diffusion in metal nanocrystal-embedded High-j/SiO2 gate stack" Appl. Phys. Lett. 99, 222102 (2011)

[78] J. Nishitani, K. Makihara, M. Ikeda, H. Murakami, S. Higashi, S. Miyazaki "Decay characteristics of electronic charged states of Si quantum dots as evaluated by an AFM/Kelvin probe technique" Thin Solid Films 508190 (2006)

[79] J. Xu, Y. Wu, W. Li and J. Xu « Surface potential modeling and reconstruction in Kelvin probe force microscopy » Nanotechnology 28, 365705 (2017)

[80] I Alhossen, C Villeneuve-Faure, F Baudoin, F Bugarin and S Segonds "Sensitivity analysis of the electrostatic force distance curve using Sobol's method and design of experiments" J. Phys. D: Appl. Phys. 50, 035304 (2017)

[81] P. Girard "Electrostatic force microscopy: principles and some applications to semiconductors" Nanotechnology 12, 485 (2001)

[82] N.B. Bercu, L. Giraudet, O. Simonetti and M. Molinari "Development of an improved Kelvin probe force microscope for accurate local potential measurements on biased electronic devices" J. Microscopy, 267, 272 (2017)

[83] F. Mortreuil, C. Villeneuve-Faure, L. Boudou, K. Makasheva and G.Teyssedre "Charges injection phenomena at metal/dielectric interface investigated by Kelvin Probe Force Microscopy" J. Phys. D 50, 175302 (2017)

[84] G. Lubarsky, R. Shikler, N. Ashkenasy, and Y. Rosenwaks "Quantitative evaluation of local charge trapping in dielectric stacked gate structures using Kelvin probe force microscopy" Appl. Phys. Lett. 58, 2921 (1991)

[85] K. Faliya, H Kliem and C.J. Dias, " Space charge measurements with Kelvin Probe Force Microscopy” IEEE Trans. Dielec. Electr. Insul. 24, 1913 (2017) 
[86] A. Savitzky und M. J. Golay "Smoothing and differentiation of data by simplified least squares procedures" Analytical Chemistry 36, 1627 (1964)

[87] C. Villeneuve-Faure, L. Boudou, K. Makasheva and G.Teyssedre "Methodology for extraction of space charge density profiles at nanoscale from Kelvin Probe Force Microscopy measurements" Nanotechnology 28, 505701 (2017)

[88] B. Martin and H. Kliem "Transient formation of internal potentials in solid electrolytes » J. Appl. Phys. 107, 076103 (2010)

[89] A. K. Henning and T. Hochwitz "Two- dimensional surface dopant profiling in silicon using scanning Kelvin probe microscopy” J. Appl. Phys. 77, 1888 (1995)

[90] O. Kryvchenkova, I. Abdullah, J. E. Macdonald, M. Elliott, T. D. Anthopoulos, Y.-H. Lin, P. Igic, K. Kalna and R. J. Cobley "Nondestructive Method for Mapping Metal Contact Diffusion in In2O3 Thin-Film Transistors" ACS Appl. Mater. Interfaces 2016, 8, 25631-25636

[91] T. Okamoto, S. Kitagawa, N. Inoue, A. Ando "Electric field concentration in the vicinity of the interface between anode and degraded $\mathrm{BaTiO} 3$-based ceramics in multilayer ceramic capacitor" Appl. Phys. Lett. 98, 072905 (2011)

[92] R. Eisenberg, D. G. Nocera, "Preface: overview of the forum on solar and renewable energy" Inorg. Chem. 44, 6799 (2005)

[93] S.-Y. Park, R. Elbersen, J. Huskens, H. Gardeniers, J.-Y. Lee, G. Mul and J. Heo "Characterization of opto-electrical enhancement of tandem photoelectrochemical cells by using photoconductive-AFM” Nanotechnology 28, 295401 (2017)

[94] J. L. Luria, N. Hoepker, R. Bruce, A. R. Jacobs, C. Groves and J.A. Marohn "Spectroscopic Imaging of Photopotentials and Photoinduced Potential Fluctuations in a Bulk Heterojunction Solar Cell Film » ACS Nano 6, 9392 (2012)

[95] E. J. Spadafora, R. Demadrille, B. Ratier and B. Grevin "Imaging the Carrier Photogeneration in Nanoscale Phase Segregated Organic Heterojunctions by Kelvin Probe Force Microscopy" Nano Lett. 10,3337 (2010)

[96] K. Maturova, M. Kemerink, M. M. Wienk, D. S. H. Charrier, and A. J. Janssen, "Scanning Kelvin Probe Microscopy on Bulk Heterojunction Polymer Blends" Adv. Funct. Mater. 19, 1379-1386 (2009)

[97] D. A. Kamkar, M. Wang, Fred Wudl and T-Q Nguyen "Single Nanowire OPV Properties of a Fullerene-Capped P3HT Dyad Investigated Using Conductive and Photoconductive AFM" ACS Nano 6,1149 (2012)

[98] D. C. Coffey, O. G. Reid, D. B. Rodovsky, G. P. Bartholomew, and D. S. Ginger "Mapping Local Photocurrents in Polymer/Fullerene Solar Cells with Photoconductive Atomic Force Microscopy" Nanoletters 7, 738 (2008)

[99] O. Douhéret, A. Swinnen, M. Breselge, I. Van Severen, L. Lutsen, D. Vanderzande and J. Manca "High resolution electrical characterisation of organic photovoltaic blends" Micro Eng 84, 431(2007)

[100] B. J. Leever and M. F. Durstock "Spatially resolved photocurrent mapping of operating organic photovoltaic devices using atomic force photovoltaic microscopy" Appl. Phys. Lett. 92, 013302 (2008)

[101] I. Beinik, M. Kratzer, A. Wachauer, L. Wang, Y. P. Piryatinski, G. Brauer, X. Y. Chen, Y. F. Hsu, A. B. Djurišić and C. Teichert "Photoresponse from single upright-standing ZnO nanorods explored by photoconductive AFM" Beilstein J. Nanotechnol. 4, 208 (2018)

[102] M. Dante, J. Peet and T.Q. Nguyen « Nanoscale charge transport and internal structure of bulk heterojunction conjugated polymer/fullerene solar cells by Scanning Probe Microscopy" J. Phys. Chem 112, 7241 (2008) 
[103] O. G. Reid, K. Munechika, and D. S. Ginger "Space Charge Limited Current Measurements on Conjugated Polymer Films using Conductive Atomic Force Microscopy" Nanoletters 8, 1602 (2008)

[104] T. G. Ruskell, R. K. Workman, D. Chen, D. Sarid, S. Dahl, and S. Gilbert "High resolution Fowler

Nordheim field emission maps of thin silicon oxide layers" Appl. Phys. Lett. 68, 1063 (1996).

[105] U. Celano, T. Hantschel, G. Giammaria, R. Chintala, T. Conard, H. Bender and W. Vandervorst "Evaluation of the electrical contact area in contact-mode scanning probe microscopy" J. Appl. Phys. 117,214305 (2015)

[106] D. Moerman, N. Sebaihi, S. E. Kaviyil, P. Leclere, R. Lazzaroni and O. Douheret "Towards a unified description of the charge transport mechanisms in conductive atomic force microscopy studies of semiconducting polymers" Nanoscale 6, 10596 (2014)

[107] D. C. Coffey and D. S. Ginger "Time-resolved electrostatic force microscopy of polymer solar cells" Nature Material 5, 735 (2006)

[108] C. Villeneuve-Faure, D. Le Borgne, E. Bedel-Pereira, K. I. Moineau Chane-Ching, D. Hernandez-Maldonado and I. Seguy "Cross Kelvin force microscopy and conductive atomic force microscopy studies of organic bulk heterojunction blends for local morphology and electrical behavior analysis" J. Appl. Phys. 117, 055501 (2015) 